\title{
Mejora del cumplimiento de fechas programadas en servicios de calibración aplicando Seis Sigma
}

\begin{abstract}
RESUMEN
El presente artículo es el resultado de la aplicación de la metodología Seis Sigma para la mejora del cumplimiento de las fechas programadas en los servicios que se realizan en las instalaciones del cliente por parte de un laboratorio de calibración. La metodología Seis Sigma, a través de sus cinco etapas, permite definir el problema de manera específica y cuantitativa, medir el desempeño del proceso afectado, identificar y analizar las causas raíz, así como establecer las acciones para superarlas y los controles para el mantenimiento de las mejoras. De esta forma, el laboratorio incrementa el cumplimiento de las fechas programadas de un $56.2 \%$ a un $70.3 \%$, al igual que el grado de satisfacción del cliente de un $72.3 \%$ a $84.6 \%$.
\end{abstract}

Palabras clave: Seis Sigma; cumplimiento; servicio.

\section{INTRODUCCIÓN}

De acuerdo con la Organización Internacional de Normalización (2015), cuando una organización apunta hacia la calidad, se fomenta una cultura enfocada en cumplir con las necesidades y expectativas del cliente, por medio de comportamientos, actitudes, actividades y procesos que proporcionan valor; por lo tanto, la calidad del producto o del servicio ofrecido por la organización se establece con la capacidad de esta para satisfacer a los clientes. Del mismo modo, Besterfield (2009) afirma que "una organización debe proporcionar un producto o servicio de alta calidad a sus clientes, que satisfaga sus necesidades: un precio razonable, entrega oportuna y servicio excepcional" (p. 38). Por otra parte, para Gryna (2002), la satisfacción de los clientes supone el grado que estos consideran que sus expectativas son respondidas o excedidas por los beneficios recibidos. Asimismo, Quijano (2003) sostiene que, en la evaluación realizada al servicio, el cliente cree que el cumplimiento de lo pactado y la capacidad de respuesta son los factores más importantes; pues, si la empresa cumple con todas las promesas establecidas por el servicio, entonces el cliente puede confiar en ella para cualquier necesidad futura con la certeza de que no perderá tiempo ni dinero.

En efecto, Zemke (como se citó en Evans y Lindsay, 2008) menciona que los dos componentes fundamentales de la calidad del servicio son las personas y la tecnología, puesto que se ha demostrado que la satisfacción de un empleado de servicio es correlativa a la del cliente. Con respecto al otro componente, la tecnología de la información es primordial debido al manejo de la gran cantidad de información que se debe procesar y a la exigencia de los clientes por un servicio con mayor velocidad. En cambio, para Nakhai y Neves (2009), el servicio de calidad es una prioridad para aquella empresa que desea diferenciarse de su competencia.

1 Ingeniería Química por la Universidad Nacional Mayor de San Marcos (Lima, Perú). Consultor independiente (Lima, Perú).

ORCID: https://orcid.org/0000-0002-5860-4515

E-mail: zenaida.enciso@gmail.com 
Por otro lado, Lankford (2005) opina que la programación brinda una ventaja competitiva y que la búsqueda de cumplir con entregas a tiempo incentiva a tener una mejor programación. Como beneficio adicional de la programación está el desarrollo de la comunicación dentro de la organización, que se evidencia al compartir información confiable y objetiva al personal involucrado. Es por esto que, para Pérez (2014), los problemas de una organización se producen por el uso inadecuado de los recursos, ignorando los aspectos o factores que pueden favorecer el buen desempeño; por lo que se debe tomar en cuenta también el compromiso del personal, dado que este representa el factor humano que administra los demás recursos. Según Gutiérrez (2008), para que las organizaciones mejoren su desempeño y la forma para afrontar estos retos es necesario que se identifiquen las causas de fondo, las cuales muchas veces están en el diseño de los procesos.

Como han señalado otras investigaciones, problemas de incumplimiento de la fecha de compromiso acordada con el cliente, lo cual puede suceder en un laboratorio de ensayo que ofrece servicios de análisis de muestras medioambientales, se presentan en empresas de servicios y producción (Palacio, Quispe, Ylesca, Cañi y Velazco , 2013); en la industria plástica dedicada a diseñar, fabricar y comercializar materiales de empaque (Rojas, 2008); en Danuser Machine Company (Lankford, 2005); en la empresa Perú Courier (Ramos, 2013); y en empresas que fabrican diversos productos ubicados en la India (Desai, 2006).

El Seis Sigma, de acuerdo con Oke (2007), es una herramienta que se utiliza para resolver problemas organizacionales, especialmente aquellos que buscan mejorar la calidad de los servicios al cliente, lo que permite incrementar su satisfacción (Kuei y Madu, como se citó en Desai, 2006). Por ello, la presente investigación se enfoca en determinar cómo impacta la aplicación de la metodología Seis Sigma en la atención de los servicios de calibración realizados en campo y en la ciudad de Lima.

\section{METODOLOGÍA}

\section{Tipo de investigación}

La investigación es aplicada, puesto que, según la definición de Sánchez y Reyes (2015), este tipo de estudio se interesa en aplicar los conocimientos teóricos a una determinada situación y en las consecuencias prácticas que resulten; además, su objetivo es conocer para hacer, actuar y modificar alguna situación. Entonces, en esta investigación, la finalidad de aplicar la metodología Seis Sigma fue para cambiar el resultado del cumplimiento de las fechas programadas de los servicios que se realizan en las instalaciones del cliente.

Asimismo, la investigación es descriptiva, porque busca explicar propiedades, características y rasgos importantes de cualquier fenómeno que se analice. Es decir, únicamente se pretende recoger información sobre los conceptos o las variables con las que se trabajan, de manera independiente o conjunta, como lo señalan Hernández, Fernández y Baptista (2010). Por tanto, en el trabajo se buscó recopilar y medir la información de las variables que afectaron el cumplimiento de las fechas programadas de los servicios que se realizan en las instalaciones del cliente.

\section{Diseño de la investigación}

El diseño de la investigación es cuasiexperimental, debido a que no hubo control total sobre las condiciones experimentales, ni la capacidad de seleccionar o asignar aleatoriamente los sujetos a los grupos de estudio. Del mismo modo, el diseño utilizado fue el pretest y postest, y se empleó de la siguiente manera:

$$
\mathrm{O}_{1} \mathrm{O}_{2} \mathrm{O}_{3} \mathrm{XO}_{4} \mathrm{O}_{5} \mathrm{O}_{6}
$$

Donde:

$\mathrm{X}$ : es la variable independiente; es decir, la implementación del Seis Sigma

$\mathrm{O}_{\mathrm{i}}$ : son las observaciones antes y después de la variable dependiente

\section{Unidad de análisis}

La unidad de análisis de la investigación correspondió a los servicios de calibración realizados por el laboratorio en las instalaciones del cliente.

\section{Población de estudio y tamaño de muestra}

Para este trabajo no se utilizó población de estudio ni tamaño de muestra, ya que el proyecto hizo referencia a una investigación por caso, y se analizó profundamente la unidad para responder al planteamiento del problema, probar la hipótesis y desarrollar alguna teoría. Para las encuestas, el tipo de muestreo empleado fue el tipo probabilístico estratificado.

\section{Técnica de recolección de datos}

La Tabla 1 muestra las técnicas e instrumentos empleados. 
Tabla 1. Técnicas de recolección de datos.

\begin{tabular}{|c|c|}
\hline Técnica & Instrumentos \\
\hline Cuestionarios & Registros del sistema de gestión \\
\hline Entrevistas & Esquema y toma de notas \\
\hline Revisión de gabinete & Reportes de base de datos \\
\hline Escalas & Encuestas \\
\hline
\end{tabular}

Fuente: Elaboración propia.

\section{Análisis de datos}

En la Tabla 2 se indica el proceder para el análisis de los datos.

\section{RESULTADOS}

\section{Etapa Definir}

En esta etapa se define el problema preliminar, con el objetivo de determinar el alcance del proyecto, y los factores críticos de calidad para el cliente (Molteni y Cecchi, 2005).

En el trabajo se tiene claro que el principal problema del laboratorio es el incumplimiento de las fechas programadas para la ejecución de los servicios de calibración, el cual genera pérdidas económicas $-73.4 \%$ por pérdida de clientes (servicios que se ejecutaron sin cumplir con la fecha), $12 \%$ por sobrecostos y $14.6 \%$ por servicios anulados-. Esto se refleja en los resultados de las encuestas de satisfacción, donde el menor nivel de satisfacción se encuentra en el primer ítem, el cual tiene un $72.3 \%$. Por otra parte, las quejas muestran que el $24.4 \%$ de estas están relacionadas con la demora en la atención de los servicios; asimismo, con respecto a la medición del proceso, se tiene establecido un cumplimiento de las fechas programadas de atención del servicio de $80 \%$ como mínimo, pero se cumple con el $56.2 \%$.

Por tanto, se busca incrementar el porcentaje del cumplimiento de las fechas programadas a un $80 \%$ y, por ende, el grado de satisfacción del cliente. Para conseguirlo, los procesos están relacionados con la programación del servicio en campo y la calibración de equipos de medición, debido a que en el primero se programa y en el segundo se ejecuta propiamente el servicio. Cabe mencionar que estos tienen subprocesos, como el que se muestra en la Figura 1.

Los resultados de las encuestas de satisfacción del cliente, las quejas y los comentarios del cliente, por medio del personal del laboratorio y de una investigación interna, permiten conocer la voz del cliente y sus requerimientos (ver Tabla 3), así como el efecto de no tomar acciones sobre la opinión del cliente.

\section{Etapa Medir}

En esta etapa se evalúan las características de los procesos identificados en la primera etapa, a través de la estratificación de factores y del nivel de desempeño de los indicadores definidos, de acuerdo con lo planteado por Molteni y Cecchi (2005). Además, se observa que:

- El cumplimiento de las fechas programadas de los servicios es menor en provincias que en Lima. Sin embargo, el proceso para la ejecución de los servicios realizados en provincias es similar a los de Lima.

- El personal influye en el cumplimiento de las fechas programadas; por ejemplo, el

Tabla 2. Matriz de análisis de datos.

\begin{tabular}{|l|l|l|l|l|l|}
\hline \multicolumn{2}{|c|}{ Variable dependiente } & \multicolumn{1}{|c|}{ Indicador } & $\begin{array}{l}\text { Escala de } \\
\text { medición }\end{array}$ & \multicolumn{1}{|c|}{ Estadísticos descriptivos } & Análisis inferencial \\
\hline $\begin{array}{l}\text { H. } \\
\text { E. 1 }\end{array}$ & $\begin{array}{l}\text { Cumplimiento de las } \\
\text { fechas programadas de } \\
\text { ejecución de los servicios } \\
\text { de calibración realizados } \\
\text { en campo }\end{array}$ & $\begin{array}{l}\text { Ratio de cumpli- } \\
\text { miento de fechas } \\
\text { programadas }\end{array}$ & $\begin{array}{l}\text { Proporción } \\
\text { o razón } \\
(0-100)\end{array}$ & $\begin{array}{l}\text { Medidas de tendencia central: me- } \\
\text { dia aritmética, mediana y moda } \\
\text { Medidas de variabilidad: desviación } \\
\text { estándar y coeficiente de variación } \\
\text { Tabla de frecuencias }\end{array}$ & t de Student \\
\hline $\begin{array}{l}\text { H. } \\
\text { E. 2 }\end{array}$ & $\begin{array}{l}\text { Nivel de satisfacción del } \\
\text { cliente después de imple- } \\
\text { mentar la metodología Seis } \\
\text { Sigma (6S) }\end{array}$ & $\begin{array}{l}\text { Ratio de satisfacción } \\
\text { del cliente después } \\
\text { de implementar 6S }\end{array}$ & $\begin{array}{l}\text { Ordinal } \\
(1-6)\end{array}$ & Conteo & 2 proporciones \\
\hline $\begin{array}{l}\text { H. } \\
\text { E. 3 }\end{array}$ & $\begin{array}{l}\text { Nivel de quejas recibidas } \\
\text { por programación de los } \\
\text { servicios }\end{array}$ & $\begin{array}{l}\text { Ratio de quejas } \\
\text { recibidas por pro- } \\
\text { gramación de los } \\
\text { servicios después de } \\
\text { implementar 6S }\end{array}$ & $\begin{array}{l}\text { Proporción } \\
\text { orazón } \\
(0-100)\end{array}$ & $\begin{array}{l}\text { Medidas de tendencia central: me- } \\
\text { dia aritmética, mediana y moda } \\
\text { Medidas de variabilidad: desviación } \\
\text { estándar y coeficiente de variación } \\
\text { Tabla de frecuencias }\end{array}$ & t de Student \\
\hline
\end{tabular}

Fuente: Elaboración propia. 


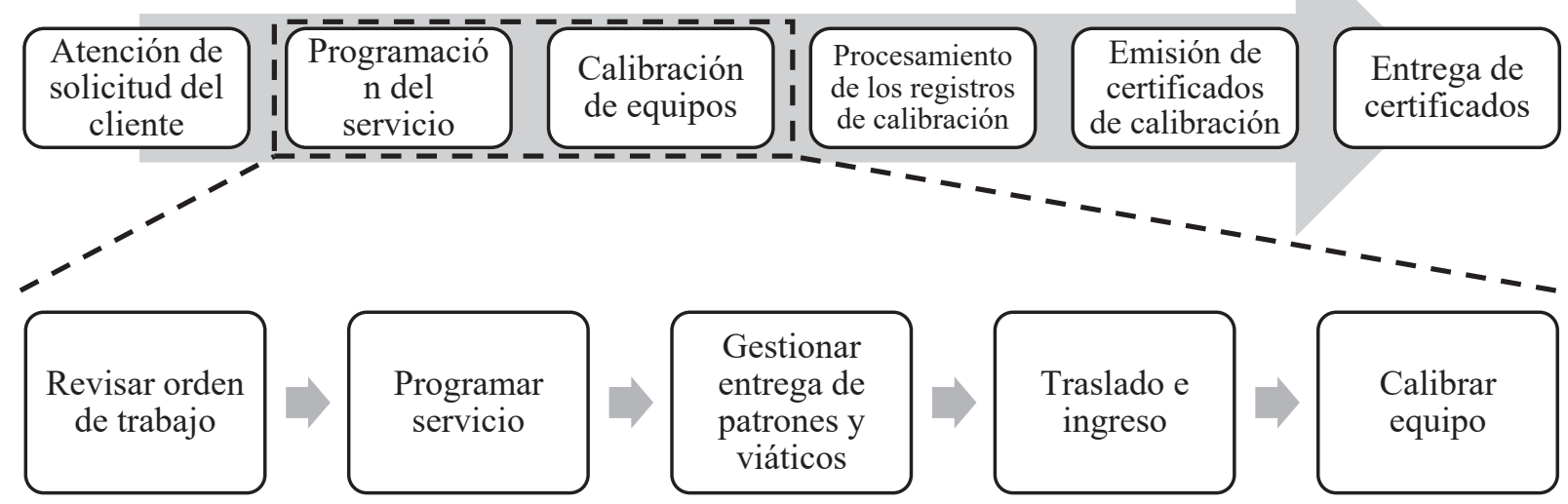

Figura 1. Procesos relacionados con la calibración de equipos.

Fuente: Elaboración propia.

Tabla 3. La voz del cliente.

\begin{tabular}{|c|c|c|}
\hline Opinión del cliente & Efecto & Requerimiento \\
\hline $\begin{array}{l}\text { No responden las llamadas o correos para consultas } \\
\text { sobre la atención de los servicios } \\
\text { Demora en la programación del servicio de calibración } \\
\text { No se solicita confirmación de la fecha programada; y, } \\
\text { si se realiza, no es con el usuario directo } \\
\text { No se cumple con la fecha y la hora programadas de } \\
\text { atención del servicio } \\
\text { El personal que inicia el servicio es distinto al que } \\
\text { termina, lo cual no se le comunica al cliente } \\
\text { No se culmina con el servicio según lo programado } \\
\text { Se reprograma el servicio a último momento } \\
\text { El técnico no cuenta con los patrones para realizar el } \\
\text { servicio }\end{array}$ & $\begin{array}{l}\text { Pérdida de clientes } \\
\text { Incremento de las quejas } \\
\text { Pérdida de imagen } \\
\text { Retrabajos } \\
\text { Disminución de las ventas } \\
\text { Publicidad negativa } \\
\text { Desmotivación del personal } \\
\text { Incremento de costos de no } \\
\text { calidad } \\
\text { Cancelación de servicios }\end{array}$ & $\begin{array}{l}\text { Accesibilidad a la empresa } \\
\text { Rápida respuesta a la programación } \\
\text { Mantener una comunicación activa entre } \\
\text { cliente-usuario para la programación de los } \\
\text { servicios } \\
\text { Atender el servicio y culminarlo según lo } \\
\text { programado } \\
\text { Comunicar posibles cambios durante la ejecu- } \\
\text { ción del servicio } \\
\text { Mantener la programación del servicio } \\
\text { Confiabilidad del servicio. } \\
\text { Calificación de los resultados presentados } \\
\text { El personal debe estar provisto con los patro- } \\
\text { nes requeridos para atender el servicio }\end{array}$ \\
\hline
\end{tabular}

Fuente: Elaboración propia.

coordinador que realiza el servicio, si bien no tiene la información completa, solicita la información al hacer las coordinaciones con el cliente. Asimismo, la cantidad de servicios a programar también es influyente, por lo que se considera a los coordinadores para el análisis.

- Los resultados del cumplimiento de las fechas responden al vendedor, quien genera la orden de trabajo y consigna toda la información respecto al servicio que se brinda. A esto se le conoce como información técnica, información del contacto-usuario, etc.

- Al ser cada procedimiento de calibración distinto para su ejecución, sobre todo en el empleo de los instrumentos de medición a emplear y en las condiciones bajo las cuales se realiza, esto afecta también en el cumplimiento de la fecha programada, tanto por la persona que debe consignar la información como por aquella que debe realizar las coordinaciones para su ejecución.

De la evaluación de desempeño del proceso (ver Figura 2) se advierte que el nivel sigma obtenido para el proceso es de 1.68 con 426205 DPMO, y con un rendimiento del $57.38 \%$. Por lo tanto, el proceso se encuentra fuera de control y no es satisfactorio.

\section{Etapa Analizar}

Molteni y Cecchi (2005) señalan que, en esta etapa, entender la relación entre las variables y los resultados de los procesos permite la identificación 


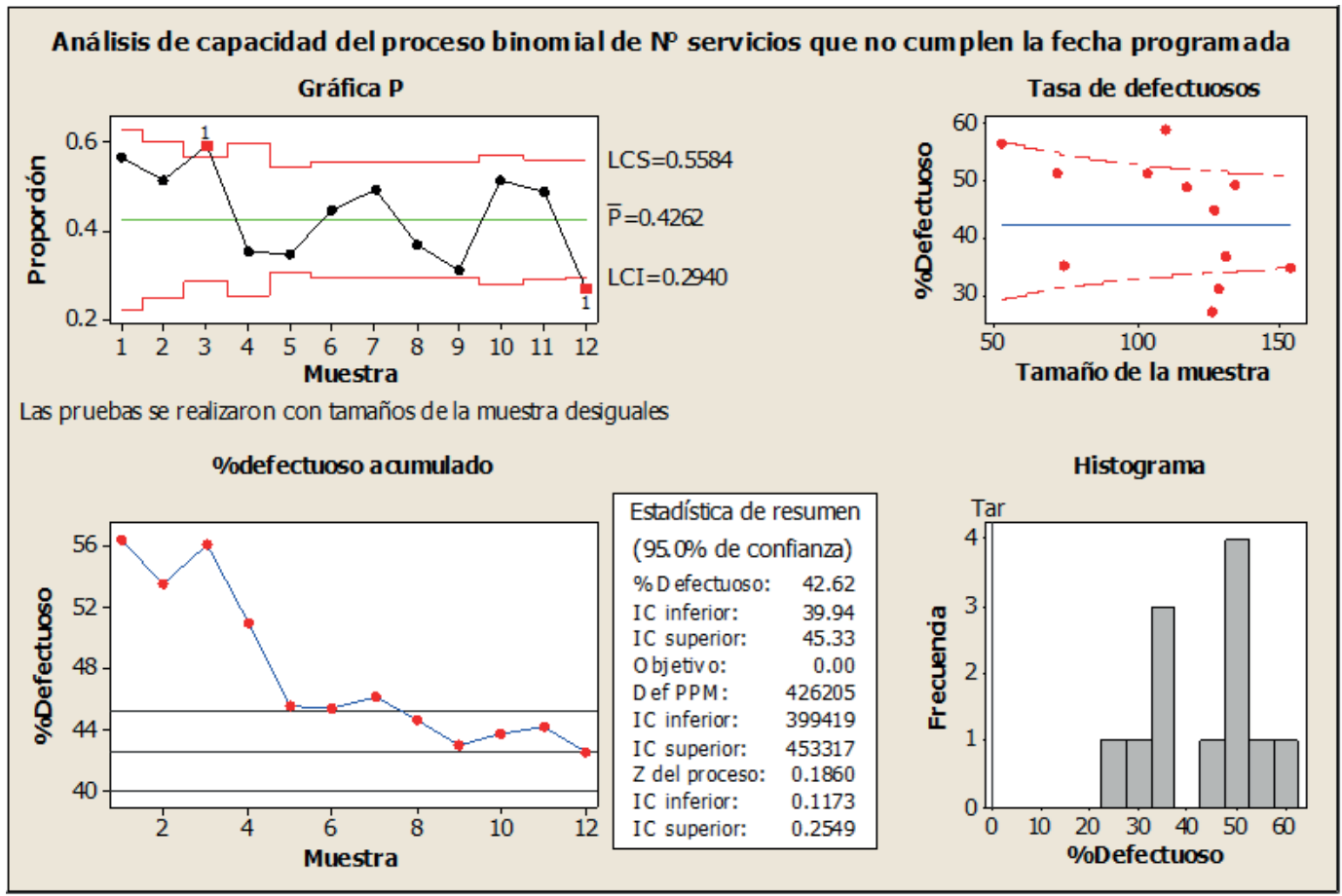

Figura 2. Análisis de la capacidad del proceso binomial del número de servicios que no cumplen con la fecha programada.

Fuente: Elaboración propia.

de su relación. Por medio del Análisis de Modo y Efecto de Fallas (AMEF) y la identificación de posibles causas del flujograma del proceso, se identifican las causas potenciales, aquellas que son sometidas a revisión por el equipo, con la finalidad de determinar las variables que se analizan estadísticamente. De la misma manera, se busca la relación causa-efecto y el grado de contribución de la variable $(X)$, para identificarlas a las de mayor significancia como causas raíz.

Para el análisis se emplea un nivel de significancia de $95 \%$ y son procesados con el software estadístico Minitab 16. A continuación, la Tabla 4 muestra el resultado del análisis de las causas raíz identificadas:

- Vendedor que genera la orden de trabajo

- Falta de comunicación con el cliente

- Coordinador que programa el servicio de calibración

\section{Etapa Mejorar}

En esta etapa se establece el diseño y la implementación de las soluciones (Molteni y Cecchi, 2005). En otras palabras, se determinan los planes de acción para mejorar el cumplimiento de las fechas programadas, tomando en cuenta la participación de un equipo multidisciplinario, dentro del cual resalta:

- El coordinador de campo, quien programa la fecha de atención del servicio, y examina la información que es recogida por la orden de trabajo y otras consideraciones dadas por el mismo cliente en la programación de la fecha de atención.

- El vendedor, quien genera la orden de trabajo y proporciona la información relacionada con el servicio a brindar.

- La falta de comunicación al cliente sobre la fecha programada de atención del servicio.

Asimismo, a fin de mantener e incrementar el cumplimiento de las fechas programadas, se establecen mecanismos a prueba de errores (Poka-Yokes), como los que se muestran en la Tabla 5:

Las variables (causa raíz) obtienen un incremento del cumplimiento de las fechas programadas, como se muestra en la Tabla 6 , aunque no significativo cuando el p-value $>0.05$. Sin embargo, se observa una disminución de la variabilidad. 
Tabla 4. Resultados del análisis y validación de las causas raíz.

\begin{tabular}{|l|l|l|l|l|l|}
\hline \multicolumn{1}{|c|}{ Variable X } & \multicolumn{1}{|c|}{ Y } & \multicolumn{1}{c|}{$\begin{array}{c}\text { Técnica } \\
\text { estadística }\end{array}$} & \multicolumn{1}{c|}{ p-value } & Significativa & \multicolumn{1}{c|}{ Validación de causa } \\
\hline $\begin{array}{l}\text { Vendedor que } \\
\text { genera orden de } \\
\text { trabajo (OT) }\end{array}$ & $\begin{array}{l}\text { Cumple fecha } \\
\text { programada }\end{array}$ & $\begin{array}{l}\text { ANOVA dos } \\
\text { factores }\end{array}$ & 0.000 & Sí & $\begin{array}{l}\text { Se realiza el análisis considerando datos quin- } \\
\text { cenales (X: vendedor; Y: cumple con la fecha } \\
\text { programada). Se observa que, al no ser todas } \\
\text { distribuciones normales, se aplica Kruskal Wallis } \\
\text { y se encuentra que las medias son significativa- } \\
\text { programada diferentes (p-value de 0.000) }\end{array}$ \\
\cline { 2 - 7 } & $\begin{array}{l}\text { ANOVA un solo } \\
\text { factor }\end{array}$ & 0.000 & Sí & $\begin{array}{l}\text { Con el estadístico t-2 muestras para la falta y no } \\
\text { de comunicación con el cliente, se obtiene un } \\
\text { p-value de 0.014 y se concluye que afecta }\end{array}$ \\
\hline $\begin{array}{l}\text { Falta de comuni- } \\
\text { cación con cliente }\end{array}$ & $\begin{array}{l}\text { Cumple fecha } \\
\text { programada }\end{array}$ & $\begin{array}{l}\text { Correlación-re- } \\
\text { gresión }\end{array}$ & 0.000 & Sí & - \\
\hline $\begin{array}{l}\text { Persona que } \\
\text { programa }\end{array}$ & $\begin{array}{l}\text { Cumple fecha } \\
\text { programada }\end{array}$ & t-2 muestras & 0.001 & Sí & \\
\hline
\end{tabular}

Fuente: Elaboración propia.

Tabla 5. Poka-Yokes.

\begin{tabular}{|c|c|c|c|}
\hline Defectos & Errores & Tipo de función & Nivel \\
\hline $\begin{array}{l}\text { Generar la orden de trabajo sin la informa- } \\
\text { ción técnica completa }\end{array}$ & $\begin{array}{l}\text { Desconocimiento del personal de } \\
\text { ventas }\end{array}$ & Advertencia & Detecta error en el momento \\
\hline $\begin{array}{l}\text { Incumplir con el programa de autorización } \\
\text { del personal }\end{array}$ & Olvido/desconocimiento & Advertencia & Detecta error en el momento \\
\hline $\begin{array}{l}\text { Programar personal técnico que no esté } \\
\text { autorizado }\end{array}$ & Desconocimiento del coordinador & Advertencia & Detecta error en el momento \\
\hline Programar personal no disponible & Cruce de fechas por inadvertencias & Control & Detecta error en el momento \\
\hline No disponer de patrones para el servicio & Inadvertencias & Advertencia & Detecta error en el momento \\
\hline $\begin{array}{l}\text { No comunicar al cliente la fecha de progra- } \\
\text { mación del servicio }\end{array}$ & $\begin{array}{l}\text { Olvido/desconocimiento/falta de } \\
\text { estándares }\end{array}$ & Advertencia & Elimina error en la fuente \\
\hline $\begin{array}{l}\text { No tener la confirmación del servicio por } \\
\text { parte del cliente }\end{array}$ & $\begin{array}{l}\text { Olvido/desconocimiento/falta de } \\
\text { estándares }\end{array}$ & Advertencia & Elimina error en la fuente \\
\hline Demorar en la programación del servicio & Olvido/desconocimiento & Advertencia & Elimina error en la fuente \\
\hline Reprogramar el servicio & Olvido/desconocimiento & Advertencia & Elimina error en la fuente \\
\hline No ingresar fecha programada al sistema & Olvido/desconocimiento & Advertencia & Detecta error en el momento \\
\hline No comunicar al personal técnico & $\begin{array}{l}\text { Olvido/desconocimiento/falta de } \\
\text { estándares }\end{array}$ & Advertencia & Elimina error en la fuente \\
\hline
\end{tabular}

Fuente: Elaboración propia.

Tabla 6. Comparación de resultados del antes y después de las mejoras implementadas.

\begin{tabular}{|l|c|c|c|c|c|}
\hline \multirow{2}{*}{ Variable } & \multicolumn{2}{|c|}{ Antes de mejoras } & \multicolumn{2}{c|}{ Después de mejoras } & \multirow{2}{*}{ P-value } \\
\cline { 2 - 5 } & Media & $\begin{array}{c}\text { Desviación } \\
\text { estándar }\end{array}$ & Media & $\begin{array}{c}\text { Desviación } \\
\text { estándar }\end{array}$ & 0.000 \\
\hline Coordinador 1 & $57.00 \%$ & 0.0522 & $68.88 \%$ & 0.0635 & 0.091 \\
\hline Coordinador 2 & $62.3 \%$ & 0.176 & $72.14 \%$ & 0.0674 & 0.078 \\
\hline Vendedor 1 & $54.80 \%$ & 0.115 & $62.95 \%$ & 0.0602 & 0.943 \\
\hline Vendedor 2 & $62.00 \%$ & 0.165 & $62.34 \%$ & 0.0595 & 0.772 \\
\hline Vendedor 3 & $61.0 \%$ & 0.189 & $62.80 \%$ & 0.0793 & 0.040 \\
\hline Vendedor 4 & $52.0 \%$ & 0.125 & $62.56 \%$ & 0.0503 & 0.067 \\
\hline Vendedor 5 & $50.2 \%$ & 0.201 & $62.96 \%$ & 0.0959 & 0.763 \\
\hline Vendedor 6 & $57.8 \%$ & 0.343 & $60.92 \%$ & 0.0483 & 0.010 \\
\hline Comunicación con el cliente & $64.4 \%$ & 0.146 & $77.71 \%$ & 0.0492 & 0 \\
\hline
\end{tabular}

Fuente: Elaboración propia. 
Se aprecia un incremento del nivel de satisfacción de los clientes para el cumplimiento de las fechas programadas a $84.6 \%$, comparado con el $72.3 \%$ inicial.

\section{Etapa Controlar}

En esta etapa se debe asegurar la permanencia de los resultados (Molteni y Cecchi, 2005). Por esa razón, se busca que las variables de los procesos relacionados con el de programación y ejecución del servicio mantengan las mejoras obtenidas. Para ello se establecen los planes de control de la Tabla 7:

Asimismo, se realizó un cuadro comparativo de los indicadores antes y después de aplicar la metodología, cuyos resultados se muestran en la Tabla 8.

De lo anterior, se desprende que, si bien se ha mejorado en el cumplimiento de la fecha programada (70.3\%), este aún no ha sido lo suficiente para cumplir con la meta del 80\% como mínimo.

Tabla 7. Plan de control.

\begin{tabular}{|c|c|c|c|c|c|c|}
\hline $\begin{array}{l}\text { Paso del } \\
\text { proceso }\end{array}$ & Variable & ¿Crítico? & $\begin{array}{l}\text { Especificación } \\
\text { (Característica a } \\
\text { controlar) }\end{array}$ & $\begin{array}{l}\text { Criterio de } \\
\text { aceptación }\end{array}$ & $\begin{array}{l}\text { Método de } \\
\text { medición }\end{array}$ & Método de control \\
\hline \multirow{2}{*}{$\begin{array}{l}\text { Revisar } \\
\text { orden de } \\
\text { trabajo }\end{array}$} & $\begin{array}{l}\text { Información técnica } \\
\text { de la orden de } \\
\text { trabajo }\end{array}$ & Sí & \multirow{2}{*}{$\begin{array}{l}\text { Grado que las órdenes } \\
\text { de trabajo cuentan } \\
\text { con la información } \\
\text { completa del equipo a } \\
\text { calibrar }\end{array}$} & \multirow{2}{*}{$\begin{array}{l}\text { Órdenes de trabajo } \\
\text { con la información } \\
\text { técnica completa del } \\
\text { equipo a calibrar }\end{array}$} & \multirow{2}{*}{$\begin{array}{l}\text { Órdenes de } \\
\text { trabajo gene- } \\
\text { radas }\end{array}$} & \multirow{2}{*}{$\begin{array}{l}\text { Gráfica tipo } p \\
\text { Mecanismo de adver- } \\
\text { tencia para completar } \\
\text { los datos del equipo al } \\
\text { generar la propuesta }\end{array}$} \\
\hline & Personal de ventas & Sí & & & & \\
\hline \multirow{4}{*}{$\begin{array}{l}\text { Programar } \\
\text { el servicio }\end{array}$} & \multirow{2}{*}{ Planificación } & Sí & $\begin{array}{l}\text { Grado de comunica- } \\
\text { ción de la fecha de } \\
\text { atención del servicio } \\
\text { programado }\end{array}$ & $\begin{array}{l}\text { Mínimo } 75 \% \text { de los } \\
\text { servicios programa- } \\
\text { dos }\end{array}$ & $\begin{array}{l}\text { Control de } \\
\text { órdenes de } \\
\text { trabajo }\end{array}$ & $\begin{array}{l}\text { Gráfica tipo } p \\
\text { Mecanismo de } \\
\text { advertencia para la } \\
\text { comunicación de la } \\
\text { fecha al cliente }\end{array}$ \\
\hline & & Sí & $\begin{array}{l}\text { Grado en que los } \\
\text { servicios son progra- } \\
\text { mados en no más de } \\
\text { dos días de generada } \\
\text { la OT }\end{array}$ & $\begin{array}{l}\text { Mínimo } 80 \% \text { de las } \\
\text { órdenes de trabajo } \\
\text { generadas }\end{array}$ & $\begin{array}{l}\text { Control de } \\
\text { órdenes de } \\
\text { trabajo }\end{array}$ & $\begin{array}{l}\text { Gráfico tipo } p \\
\text { Sistema a prueba de } \\
\text { error }\end{array}$ \\
\hline & Personal técnico & Sí & $\begin{array}{l}\text { Cumplimiento del pro- } \\
\text { grama de autorización } \\
\text { del personal }\end{array}$ & $\begin{array}{l}\text { Personal con autori- } \\
\text { zación vigente }\end{array}$ & $\begin{array}{l}\text { Programa de } \\
\text { autorización }\end{array}$ & $\begin{array}{l}\text { Sistemas a prueba de } \\
\text { error }\end{array}$ \\
\hline & $\begin{array}{l}\text { Documentos del } \\
\text { personal técnico }\end{array}$ & No & $\begin{array}{l}\text { Mantener actualizada } \\
\text { la base de datos del } \\
\text { personal, considerando } \\
\text { la vigencia de chequeo } \\
\text { médico, inducciones, } \\
\text { charlas, etc. }\end{array}$ & $\begin{array}{l}\text { Personal técnico con } \\
\text { requerimientos del } \\
\text { cliente vigentes }\end{array}$ & $\begin{array}{l}\text { Base de } \\
\text { requerimientos } \\
\text { del personal }\end{array}$ & $\begin{array}{l}\text { Sistemas a prueba de } \\
\text { error }\end{array}$ \\
\hline \multirow{2}{*}{$\begin{array}{l}\text { Gestionar } \\
\text { entrega de } \\
\text { patrones }\end{array}$} & \multirow{2}{*}{$\begin{array}{l}\text { Patrones de me- } \\
\text { dición aptos para } \\
\text { su uso }\end{array}$} & \multirow{2}{*}{ Sí } & $\begin{array}{l}\text { Cumplimiento del pro- } \\
\text { grama de calibración } \\
\text { y verificación de los } \\
\text { patrones de medición }\end{array}$ & $\begin{array}{l}\text { Patrones calibrados } \\
\text { y verificados }\end{array}$ & $\begin{array}{l}\text { Programa de } \\
\text { calibración y } \\
\text { verificación }\end{array}$ & $\begin{array}{l}\text { Mecanismo a prueba } \\
\text { de error }\end{array}$ \\
\hline & & & $\begin{array}{l}\text { Cumplimiento del } \\
\text { programa de manteni- } \\
\text { miento de los patrones } \\
\text { de medición }\end{array}$ & $\begin{array}{l}\text { Patrones con mante- } \\
\text { nimiento y operativos }\end{array}$ & $\begin{array}{l}\text { Programa de } \\
\text { mantenimiento }\end{array}$ & $\begin{array}{l}\text { Mecanismo a prueba } \\
\text { de error }\end{array}$ \\
\hline \multirow{2}{*}{$\begin{array}{l}\text { Ejecutar el } \\
\text { servicio }\end{array}$} & $\begin{array}{l}\text { Cumplimiento de la } \\
\text { fecha programada }\end{array}$ & Sí & $\begin{array}{l}\text { Grado de cumplimien- } \\
\text { to de las fechas pro- } \\
\text { gramadas de atención } \\
\text { del servicio }\end{array}$ & $\begin{array}{l}\text { Mínimo } 85 \% \text { de ser- } \\
\text { vicios cumplidos }\end{array}$ & $\begin{array}{l}\text { Control de } \\
\text { órdenes de } \\
\text { trabajo }\end{array}$ & $\begin{array}{l}\text { Gráfica tipo p } \\
\text { Mecanismo de adver- } \\
\text { tencia }\end{array}$ \\
\hline & $\begin{array}{l}\text { Satisfacción del } \\
\text { cliente }\end{array}$ & Sí & $\begin{array}{l}\text { Resultado de muy } \\
\text { bueno en la encuesta } \\
\text { de satisfacción del } \\
\text { cliente }\end{array}$ & $\begin{array}{l}\text { Mínimo } 80 \% \text { de } \\
\text { satisfacción }\end{array}$ & $\begin{array}{l}\text { Encuestas de } \\
\text { satisfacción }\end{array}$ & Muestreo \\
\hline
\end{tabular}

Fuente: Elaboración propia. 
Tabla 8. Cuadro comparativo de antes y después de usar la metodología.

\begin{tabular}{|l|c|c|}
\hline \multicolumn{1}{|c|}{ Indicador } & Antes \\
\hline Indicadores de gestión & 0.356 & Después \\
\hline Cp & Proceso incapaz e inestable & Proceso incapaz y descentrado \\
\hline CEP y capacidad & $42.74 \%$ & $29.44 \%$ \\
\hline Porcentaje de defectuosos & 427376 & 294360 \\
\hline DPMO & 0.1831 & 0.5407 \\
\hline Z del proceso & 1.68 & 2.04 \\
\hline Nivel sigma & $56.2 \%$ & $70.3 \%$ \\
\hline Límite central-cumplimiento de la fecha programada & S/ 747 230 & S/ 463 989 \\
\hline Costos variables & & \\
\hline Indicadores de calidad & $72.3 \%$ & $84.6 \%$ \\
\hline Satisfacción del cliente-cumplimiento de la fecha programada & 31 & 10 \\
\hline N. ${ }^{\circ}$ quejas recibidas por programación & $31.4 \%$ & $19.6 \%$ \\
\hline Porcentaje de quejas por cumplimiento de fecha & & \\
\hline
\end{tabular}

Fuente: Elaboración propia.

\section{DISCUSIÓN}

Hipótesis específica 1: Impacta positivamente la aplicación de la metodología Seis Sigma en el cumplimiento de las fechas programadas de ejecución de los servicios de calibración realizados en campo. Para confirmar la hipótesis se aplicó:

$$
\begin{aligned}
& \text { Ho: } \mu 1=\mu 2 \\
& \text { H1: } \mu 1 \neq \mu 2
\end{aligned}
$$

\section{Donde:}

$\mu 1$ : La media del cumplimiento de la fecha programada de ejecución de los servicios de calibración al inicio.

2 : La media del cumplimiento de la fecha programada de ejecución de los servicios de calibración al final.

Por ello, para determinar si hubo mejora sobre esta variable, se comparó el antes y el después del cumplimiento de la fecha programada de ejecución del servicio, al aplicarse el t de dos muestras.

De la Figura 3 resulta que, debido a que el intervalo de confianza está entre <-0.2135; -0.0701>, no se encuentra el valor de referencia de 0 dentro de este intervalo de confianza y que el valor $p(0.001)<\alpha$ (0.05). Entonces, se rechaza Ho con una confianza de $95 \%$.

Por lo tanto, se concluyó que el cumplimiento de las fechas programadas de ejecución de los servicios de calibración antes y después de la aplicación de la metodología es distinto.

Hipótesis específica 2: Impacta positivamente la identificación de los factores de mayor influencia sobre el cumplimiento de las fechas programadas de ejecución del servicio en el nivel de satisfacción del cliente después de implementar la metodología Seis Sigma. Para confirmar la hipótesis se aplicó:

$$
\text { Ho: } p(1)-p(2)
$$

$$
H 1: p(1)-p(2) \neq 0
$$

Donde:

$\mathrm{p}(1)$ : Proporción de clientes insatisfechos antes de implementar las mejoras.

$\mathrm{p}(2)$ : Proporción de clientes insatisfechos después de implementar las mejoras.

Por ello, para determinar si hubo mejora sobre esta variable, se comparó el antes y después del nivel de satisfacción del cliente, al aplicarse la prueba de dos proporciones.

De la Figura 4 resulta que, debido a que el intervalo de confianza está entre <0.0242658; 0.221888>, el valor de referencia de 0 está fuera de este intervalo de confianza. Además del valor $p(0.015)<\alpha(0.05)$, se rechaza Ho con una confianza de $95 \%$.

Se concluyó que existe diferencia entre la satisfacción del cliente respecto al cumplimiento de las fechas programadas del servicio antes y después de 


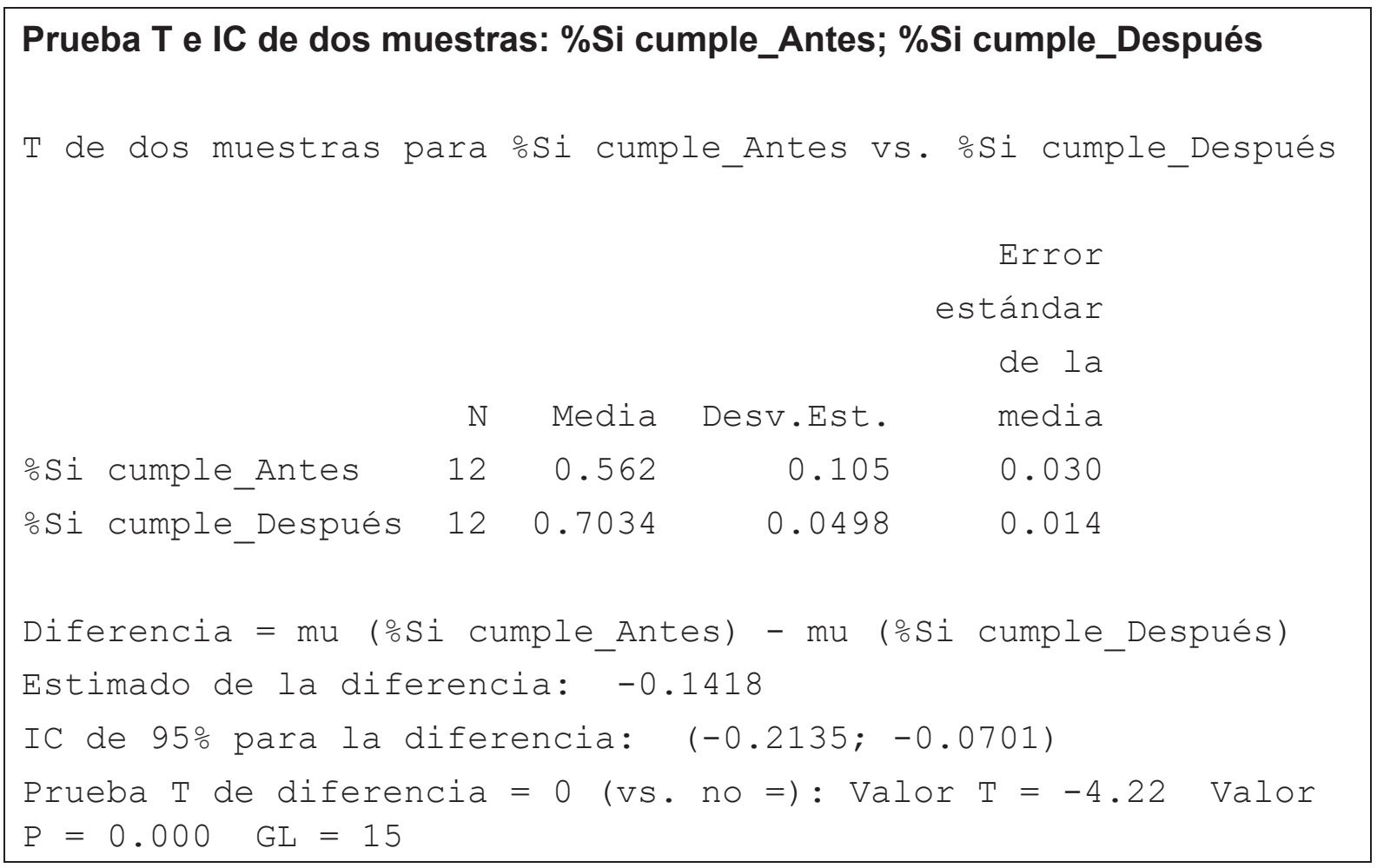

Figura 3. Prueba T e IC de dos muestras: cumplimiento de fechas programadas de la atención de servicios de calibración. Antes y después de implementar la metodología Seis Sigma.

Fuente: Elaboración propia.

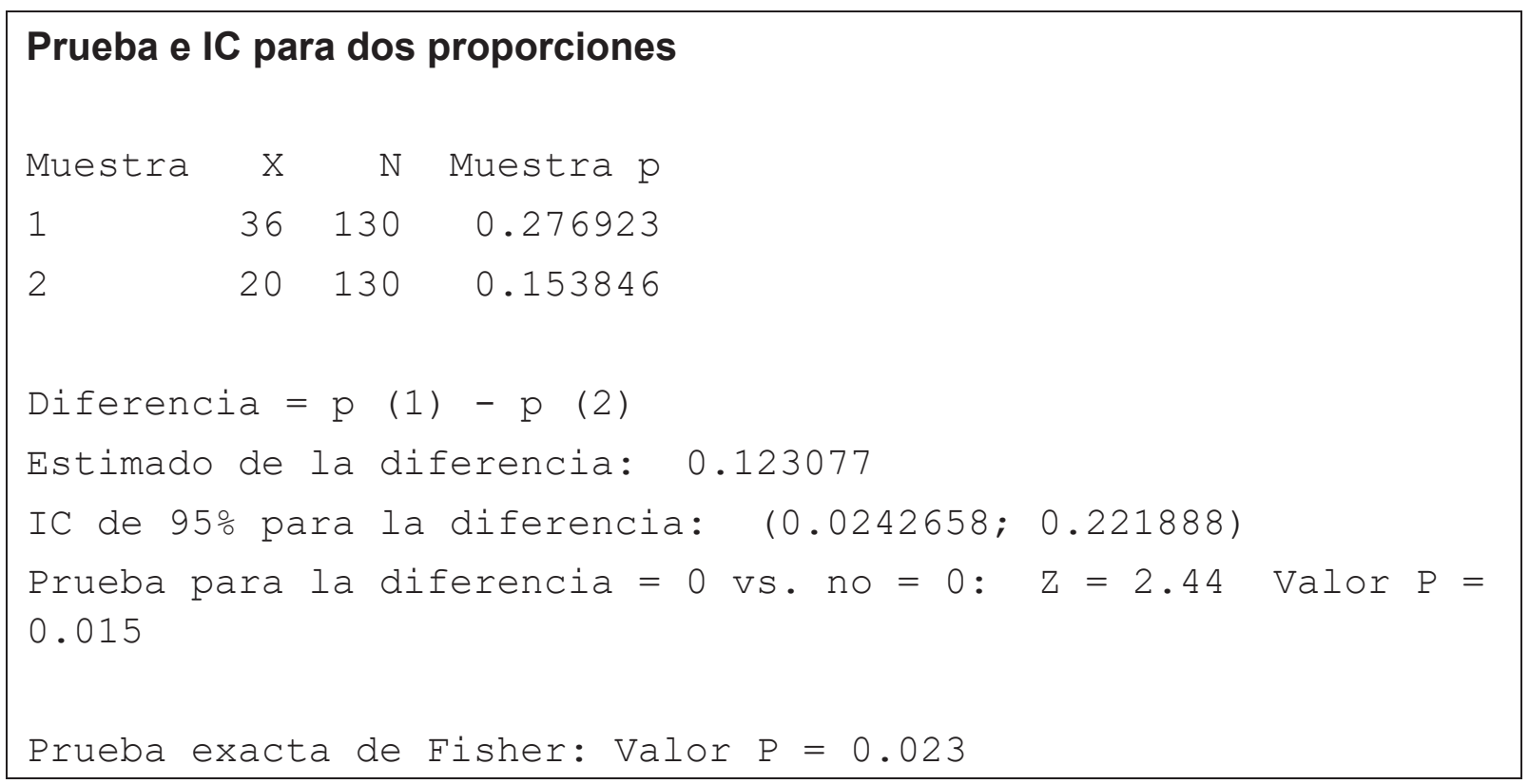

Figura 4. Prueba para dos proporciones: nivel de satisfacción del cliente para el cumplimiento de la fecha programada. Antes y después de implementar la metodología Seis Sigma. 
implementar las mejoras con la metodología Seis Sigma.

Hipótesis específica 3: Impacta positivamente la implementación de acciones sobre los factores que influyen en el cumplimiento de la fecha programada con el nivel de quejas recibidas asociado a la programación de los servicios. Para confirmar la hipótesis se aplicó:

$$
\begin{aligned}
& \text { Ho: } \mu 1=\mu 2 \\
& \text { H1: } \mu 1 \neq \mu 2
\end{aligned}
$$

Donde:

$\mu 1$ : La media de la ratio de quejas recibidas por programación de los servicios al inicio.

$\mu 2$ : La media de la ratio de quejas recibidas por programación de los servicios al final.

Por ello, para determinar si hubo mejora sobre esta variable, se comparó el antes y el después de la ratio de quejas recibidas por la programación de los servicios, al aplicarse el t de dos muestras.

De la Figura 5 resulta que, debido a que el intervalo de confianza está entre <-0.0114; 0.2470>, el valor de referencia de 0 está dentro de este intervalo de confianza. Además del valor $p(0.067)>\alpha(0.05)$, se acepta Ho con una confianza de $95 \%$.

Por lo tanto, se concluyó que la ratio de quejas recibidas por programación de servicios al iniciar el proyecto y la ratio de quejas recibidas por programación de servicios después de implementada la metodología son iguales.

\section{RECOMENDACIONES}

- Concientizar a la alta dirección de la importancia de su participación en el proyecto, con el propósito de garantizar los recursos y el apoyo gerencial e impactar en la mejora hacia la organización y, principalmente, la del cliente.

- Mantener una comunicación fluida con el personal de la organización del desarrollo del proyecto, a fin de garantizar la confianza, la participación y el compromiso hacia la mejora.

- Mantener el programa de formación para el personal involucrado, directa o indirectamente, con el proceso mejorado.

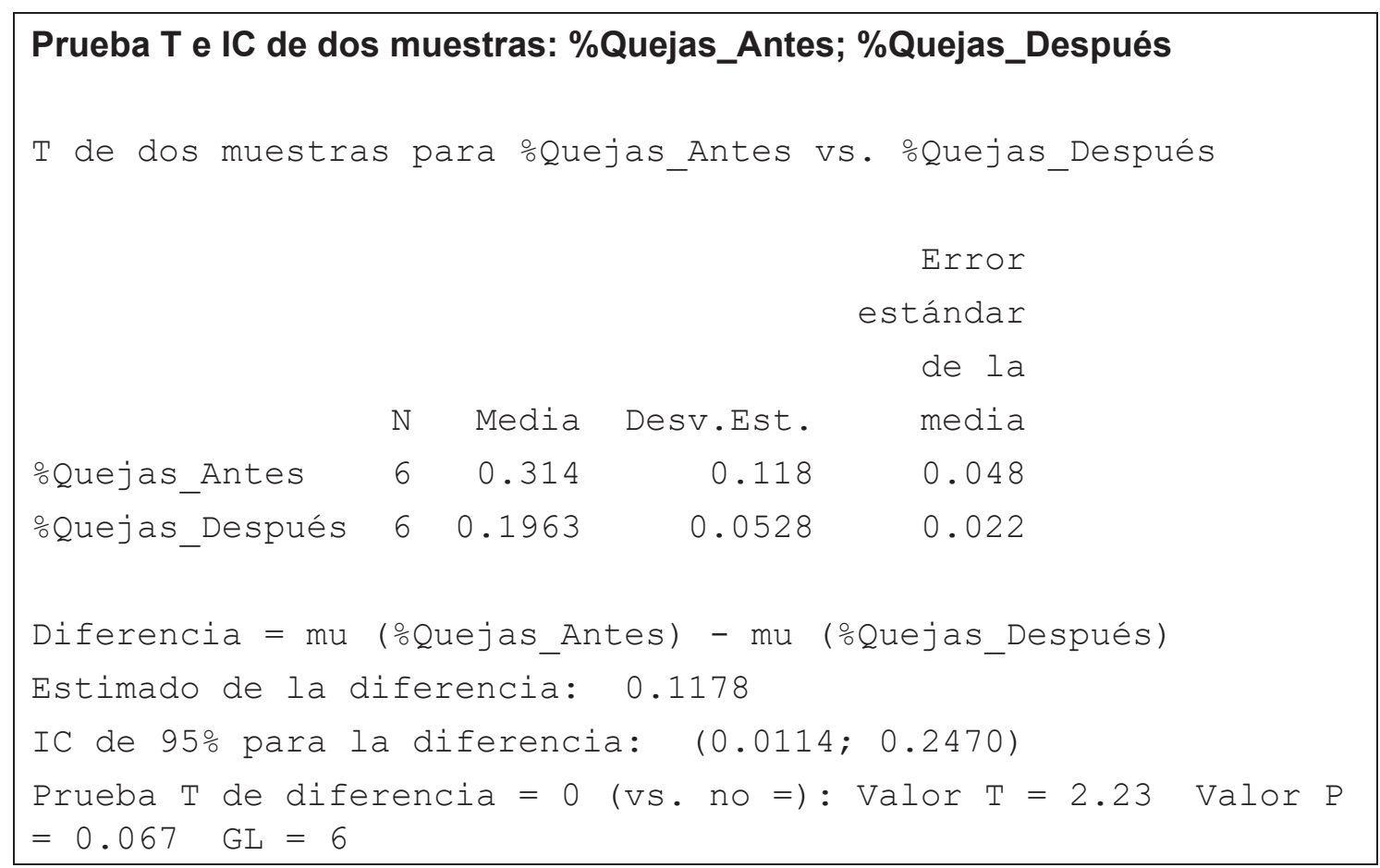

Figura 5. Prueba T e IC de dos muestras: ratio de quejas recibidas por programación de servicios. Antes y después de implementar la metodología Seis Sigma.

Fuente: Elaboración propia. 
- Emplear la metodología Seis Sigma para cumplir con la entrega de los certificados de calibración, cuyo alcance son los procesos de procesamiento de los registros de calibración, emisión de los certificados de calibración y entrega de los certificados al cliente.

\section{CONCLUSIONES}

- La aplicación de la metodología Seis Sigma impactó positivamente en el cumplimiento de las fechas programadas de ejecución de los servicios de calibración realizados en campo, al incrementar de $56.2 \%$ a $70.34 \%$.

- La identificación de los factores de mayor influencia sobre el cumplimiento de la fecha programada de ejecución de servicios impactó positivamente en el nivel de satisfacción del cliente después de implementar la metodología Seis Sigma, al incrementar de $72.3 \%$ a $84.6 \%$

- La implementación de acciones sobre los factores que influyen en el cumplimiento de la fecha programada impactó positivamente en el nivel de quejas recibidas asociado a la programación de los servicios, al disminuir de $31.4 \%$ a $19.63 \%$.

\section{REFERENCIAS BIBLIOGRÁFICAS}

[1] Besterfield, D. H. (2009). Control de calidad. México D. F., México: Pearson Educación.

[2] Desai, D. A. (2006). Improving Customer Delivery Commitments the Six Sigma Way: Case Study of an Indian Small Scale Industry. International Journal of Six Sigma and Competitive Advantage, 2(1), 23-47.

[3] Evans, J. R. y Lindsay, W. M. (2008). Administración y control de la calidad. México D. F., México: Cengage Learning.

[4] Gryna, F. M. (2002). Investigación de mercados y marketing. En J. M. Juran y A. B. Godfrey (Coords.), Manual de calidad de Juran (tomo 1) (pp. 18.1-18.38). Madrid, España: McGrawHill.

[5] Gutiérrez, H. (2008). Los retos de la mejora de la calidad y la productividad en las organizaciones. Ingeniería Industrial. Actualidad y Nuevas Tendencias, 1(1), 109-124.

[6] Hernández, R., Fernández, C. y Baptista, P. (2010). Metodología de la investigación. México D. F., México: McGraw-Hill.
[7] Lankford, R. (2005). Programación de la producción. En K. Zandin, Maynard: Manual del ingeniero industrial (pp. 9.157-9.176). México D. F., México: McGraw-Hill.

[8] Molteni, R. y Cecchi, O. (2005). El liderazgo del Lean Six Sigma. Buenos Aires, Argentina: Macchi.

[9] Nakhai, B. y Neves, J. S. (2009). The Challengues of Six Sigma in Improving Service Quality. International Journal of Quality \& Realiability Management, 26(7), 663-684.

[10] Oke, S. A. (2007). Six Sigma: A Literature Review. South African Journal of Industrial Engineering, 18(2), 109-129.

[11] Organización Internacional de Normalización (2015). Norma internacional ISO 9000. Sistemas de gestión de la calidad. Fundamentos y vocabulario. Ginebra, Suiza: Secretaría General de ISO.

[12] Palacio, V., Quispe, F., Ylesca, F., Cañi, E. y Velazco, C. (2013). Rediseño y optimización de procesos de CERPER. Lima, Perú: Instituto para la Calidad de la Pontificia Universidad Católica del Perú.

[13] Pérez, D. A. (2014). Cultura del incumplimiento empresarial en México. Ensayo. Recuperado de http://www.gestiopolis.com/cultura-delincumplimiento-empresarial-en-mexicoensayo.

[14] Quijano, V. M. (2003). Cumplimiento del servicio prometido a sus clientes. Recuperado de http://www.gestiopolis.com/cumplimientoservicio-prometido-clientes.

[15] Ramos, W. A. (2013). Incremento de la productividad a través de la mejora continua en calidad en la subunidad de procesamiento de datos en una empresa courier: el caso Perú Courier. Industrial Data, 16(2), 59-66.

[16] Rojas, L. C. (2008). Implementación del sistema de gestión de calidad según la Norma ISO 9001:2000 en una industria plástica. (Tesis de licenciatura). Escuela Superior Politécnica del Litoral, Guayaquil.

[17] Sánchez, H. y Reyes, C. (2015). Metodología y diseños en la investigación científica. Lima, Perú: Business Support Aneth S. R. L. 


\section{Improving Compliance with Scheduled Dates in Calibration Services Applying Six Sigma}

\begin{abstract}
This article is the result of the application of Six Sigma methodology to improve compliance with scheduled dates in services performed by a calibration laboratory at the customer's facilities. Through its five phases, Six Sigma methodology makes it possible to define the problem in a specific and quantitative way, measuring the performance of the affected process, identifying and analyzing the root causes, as well as establishing the actions to overcome problems and the controls to maintain the improvements. In this way, the laboratory increases the compliance with scheduled dates from $56.2 \%$ to $70.3 \%$, as well as the level of customer satisfaction from $72.3 \%$ to $84.6 \%$.
\end{abstract}

Keywords: Six Sigma; compliance; service.

\section{INTRODUCTION}

According to the International Organization for Standardization (2015), when an organization aims at quality, it promotes a culture focused on meeting customer needs and expectations through behaviors, attitudes, activities and processes that provide value; therefore, the quality of the product or service offered by the organization is established by the organization's ability to satisfy customers. Besterfield (2009) states that "an organization must give its customers a quality product or service that meets their needs at a reasonable price, which includes on-time delivery and outstanding service" (p. 38). On the other hand, for Gryna (2002), customer satisfaction is the degree to which customers consider that the benefits received meet or exceed their expectations. Similarly, Quijano (2003) argues that according to customer surveys the client believes that compliance with what was offered, and response capacity are the most important factors because if the company fulfills all the promises established by the service, the client can trust it for any future needs with the certainty that they will not lose time or money.

Indeed, Zemke (as quoted in Evans \& Lindsay, 2008) mentions that the two fundamental components of service quality are people and technology. It has been shown that service employee job satisfaction is correlated to customer satisfaction. As for the other component, information technology is paramount due to the handling of a high volume of information that needs to be processed and customers' demand for a faster service. On the other hand, for Nakhai and Neves (2009), quality service is a priority for companies that wish to differentiate themselves from their competitors.

Chemical engineer from the Universidad Nacional Mayor de San Marcos (Lima, Perú). Currently working as an independent consultant (Lima, Peru) ORCID: https://orcid.org/0000-0002-5860-4515 E-mail: zenaida.enciso@.gmail.com 
Alternatively, Lankford (2005) is of the opinion that scheduling provides a competitive advantage and that the search for timely delivery encourages better scheduling. An additional benefit of scheduling is the development of communication within the organization, which is evidenced by the sharing of reliable and objective information to the staff involved. For this reason, Pérez (2014) argues that the problems of an organization are produced by the inadequate use of resources, ignoring the aspects or factors that can favor good performance; therefore, staff commitment must also be considered, since they represent the human factor that manages the other resources. According to Gutiérrez (2008), for organizations to improve their performance and the way they face these challenges, it is necessary to identify the underlying causes, which frequently can be found in the process design.

As other investigations have pointed out, problems of non-compliance with the commitment date agreed with the client-which can happen in a testing laboratory that offers services for the analysis of environmental samples-occur in service and production companies (Palacio, Quispe, Ylesca, Cañi \& Velazco, 2013); in the plastic industry dedicated to designing, manufacturing and trading packaging materials (Rojas, 2008); in the Danuser Machine Company (Lankford, 2005); in Peru Courier (Ramos, 2013); and in companies that manufacture various products located in India (Desai, 2006).

According to Oke (2007), Six Sigma is a tool used to solve organizational problems, especially those that are related to improving the quality of customer services, thus increasing customer satisfaction (Kuei \& Madu, as quoted in Desai, 2006). Therefore, this research focuses on determining how the application of Six Sigma impacts the attention of the calibration services performed in the field, in the city of Lima.

\section{METHODOLOGY}

\section{Type of research}

This is an applied research project, since, according to the definition of Sánchez and Reyes (2015), this type of study is interested in applying theoretical knowledge to a certain situation and the practical consequences that result; furthermore, its objective is to know in order to act and modify some situation. Therefore, the purpose of applying the Six Sigma methodology was to change the result of the fulfiIlment of services scheduled dates that are performed at the client's facilities.
Likewise, the research is descriptive because it seeks to explain properties, characteristics and important features of any phenomenon analyzed. In other words, it is only intended to collect information on the concepts or variables with which it works, independently or jointly, as pointed out by Hernández, Fernández and Baptista (2010). Therefore, the work sought to collect and measure information of the variables that affected compliance with the scheduled dates of the services performed at the customer's facilities.

\section{Research design}

The research design is quasi-experimental, since there was no total control over the experimental conditions, nor the ability to select or randomly assign subjects to the study groups. Similarly, pre-test/ post-test design was used in this research, and was employed as follows:

$$
\mathrm{O}_{1} \mathrm{O}_{2} \mathrm{O}_{3} \mathrm{XO}_{4} \mathrm{O}_{5} \mathrm{O}_{6}
$$

Where:

$\mathrm{X}$ : is the independent variable; that is, Six Sigma implementation

$\mathrm{O}_{\mathrm{i}}$ : are the observations before and after the dependent variable

\section{Analysis unit}

The research analysis unit corresponded to the calibration services performed by the laboratory at the customer's facilities.

\section{Study population and sample size}

No study population or sample size was used for this research, as it referred to a case study and the unit was analyzed in depth to respond to the problem statement, test the hypothesis and develop any theory. For the surveys, stratified sampling was used.

\section{Data collection technique}

Table 1 shows the techniques and instruments used.

Table 1. Data collection techniques

\begin{tabular}{|c|c|}
\hline Techniques & Instruments \\
\hline Questionnaires & Management system records \\
\hline Interviews & Outline and note taking \\
\hline Desk research & Database reports \\
\hline Scales & Surveys \\
\hline
\end{tabular}

Source: Prepared by the author 


\section{Data analysis}

Table 2 indicates the procedure for data analysis.

\section{RESULTS}

\section{Define phase}

At this phase the preliminary problem is defined, aiming to determine the scope of the project and the critical quality factors for the client (Molteni \& Cecchi, 2005).

In this research there is no doubt that the laboratory's main problem is noncompliance with the scheduled dates for the execution of calibration services, which generates economic losses- $73.4 \%$ due to the loss of clients (services executed out of date), $12 \%$ due to cost overruns and $14.6 \%$ due to cancelled services-. This is reflected in the results of the satisfaction surveys, where the lowest level of satisfaction is found in the first item, $72.3 \%$. On the other hand, $24.4 \%$ of complaints are related to delays in the provision of services; likewise, with respect to the measurement of the process, $80 \%$ compliance with the scheduled dates for the provision of service has been established as the minimum, but is only met $56.2 \%$ of the time.

Therefore, the aim is to increase the percentage of compliance with scheduled dates to $80 \%$ and, thereby, the level of customer satisfaction. To achieve this, the processes are related to the scheduling of field service and the calibration of measuring equipment, as in the first process the service is scheduled and in the second, the service itself is executed. It is worth mentioning that these have sub-processes, such as the ones shown in Figure 1.

The results of customer satisfaction surveys, complaints and customer feedback, obtained through laboratory staff and internal research, provide insight into the voice of the customer and their requirements (see Table 3), as well as the effect of not taking action on the customer's opinion.

\section{Measure phase}

In this phase, the characteristics of the processes identified in the first phase are evaluated through the stratification of factors and the level of performance of the defined indicators, in accordance with what was proposed by Molteni and Cecchi (2005). In addition, it is observed that:

- Compliance with scheduled service dates is lower in provincial areas than in Lima. However, the process for the execution of the services provided in provincial areas is similar to those in Lima.

- Staff influence the compliance with scheduled dates; for example, the coordinator who performs the service; if the information is not complete, they request it when making the arrangements with the client. The same happens to the number of services to be scheduled, so coordinators are considered for analysis.

- The results of scheduling compliance correspond to the seller, who creates the work order and records all the information with respect to the service that is offered. This

Table 2. Data Analysis Matrix.

\begin{tabular}{|l|l|l|l|l|l|}
\hline \multicolumn{2}{|c|}{ Dependent variable } & \multicolumn{1}{|c|}{ Indicator } & $\begin{array}{c}\text { Scale of } \\
\text { Measurement }\end{array}$ & \multicolumn{1}{|c|}{ Descriptive statistics } & $\begin{array}{c}\text { Inferential } \\
\text { Analysis }\end{array}$ \\
\hline $\begin{array}{l}\text { S. } \\
\text { H. 1 }\end{array}$ & $\begin{array}{l}\text { Compliance with scheduled } \\
\text { dates for calibration ser- } \\
\text { vices performed in the field }\end{array}$ & $\begin{array}{l}\text { Scheduled dates compli- } \\
\text { ance ratio }\end{array}$ & $\begin{array}{l}\text { Proportion or } \\
\text { ratio } \\
(0-100)\end{array}$ & $\begin{array}{l}\text { Measures of central tendency: } \\
\text { arithmetic mean, median and mode } \\
\text { Measures of variability: standard } \\
\text { deviation and coefficient of variation } \\
\text { Frequency table }\end{array}$ & Student's t \\
\hline $\begin{array}{l}\text { S. } \\
\text { H. 2 }\end{array}$ & $\begin{array}{l}\text { Level of customer satisfac- } \\
\text { tion after implementing Six } \\
\text { Sigma (6S) methodology }\end{array}$ & $\begin{array}{l}\text { Ratio of customer sat- } \\
\text { isfaction after imple- } \\
\text { menting Six Sigma (6S) } \\
\text { methodology }\end{array}$ & $\begin{array}{l}\text { Ordinal } \\
(1-6)\end{array}$ & Counting & 2 proportions \\
\hline $\begin{array}{l}\text { S. } \\
\text { H. 3 }\end{array}$ & $\begin{array}{l}\text { Level of complaints } \\
\text { received for the services } \\
\text { scheduling }\end{array}$ & $\begin{array}{l}\text { Ratio of complaints } \\
\text { received for the services } \\
\text { scheduling after imple- } \\
\text { menting 6S }\end{array}$ & $\begin{array}{l}\text { Proportion or } \\
\text { ratio } \\
(0-100)\end{array}$ & $\begin{array}{l}\text { Measures of central tendency: } \\
\text { arithmetic mean, median and mode } \\
\text { Measures of variability: standard } \\
\text { deviation and coefficient of variation } \\
\text { Frequency table }\end{array}$ & Student's t \\
\hline
\end{tabular}

Source: Prepared by the author 


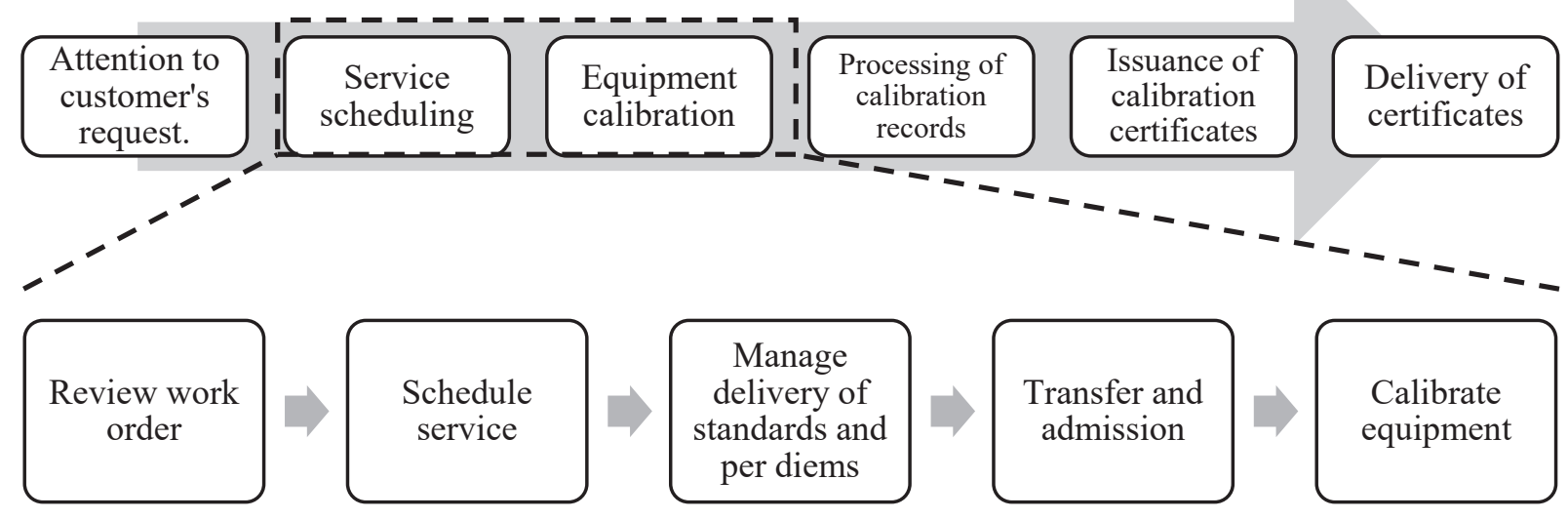

Figure 1. Processes related to equipment calibration. Source: Prepared by the author

Table 3. Voice of customer.

\begin{tabular}{|c|c|c|}
\hline inion & Effect & nent \\
\hline $\begin{array}{l}\text { Calls or emails with inquiries about the services are } \\
\text { not answered } \\
\text { Delay in scheduling calibration service } \\
\text { No confirmation of the scheduled date is requested; } \\
\text { and, if it is, it is not with the actual user } \\
\text { Scheduled date and time of service is not met } \\
\text { The staff that starts the service is different from the } \\
\text { one that finishes, the client is not informed of this } \\
\text { change } \\
\text { Failure to complete service as scheduled } \\
\text { Service is rescheduled at the last minute } \\
\text { The technician does not have the calibration stan- } \\
\text { dards to perform the service }\end{array}$ & $\begin{array}{l}\text { Loss of customers } \\
\text { Increase in complaints } \\
\text { Loss of image } \\
\text { Reworks } \\
\text { Decrease in sales } \\
\text { Negative publicity } \\
\text { Employee demotivation } \\
\text { Increasing non-quality costs } \\
\text { Cancellation of services }\end{array}$ & $\begin{array}{l}\text { Accessibility to the company } \\
\text { Rapid response to scheduling } \\
\text { Maintaining active communication between custo- } \\
\text { mer and user for the services scheduling } \\
\text { Execute the service and complete it as scheduled } \\
\text { Communicate possible changes during the exe- } \\
\text { cution of the service } \\
\text { Maintain the service scheduling } \\
\text { Service reliability } \\
\text { Qualification of the results presented } \\
\text { The staff must be given the required calibration } \\
\text { standards to execute the service }\end{array}$ \\
\hline
\end{tabular}

Source: Prepared by the author

is known as technical information, contact or user information, etc.

- As each calibration procedure is different in its execution, especially in the use of the measuring instruments to be used and in the conditions under which it is performed, compliance with the scheduled date is affected both by the person who must record the information and by the person who must make the arrangements for its execution.

From the process performance evaluation (see Figure 2) it can be seen that the sigma level obtained for the process is 1.68 with 426205 DPMO, and with a yield of $57.38 \%$. Therefore, the process is not under control and is not satisfactory.

\section{Analyze phase}

Molteni and Cecchi (2005) point out that, at this phase, understanding the relationship between the variables and the results of the processes is necessary in order to identify their relationship. By means of Failure Mode and Effects Analysis (FMEA) and the identification of possible causes of the process flowchart, potential causes are identified, which are subject to review by the team, in order to determine which variables are statistically analyzed. In the same way, the cause and effect relationship and the degree of contribution of the variable $(X)$ are sought, in order to identify those of greater significance as root causes.

A significance level of $95 \%$ is used for the analysis and the data are processed with Minitab 16 statistical 


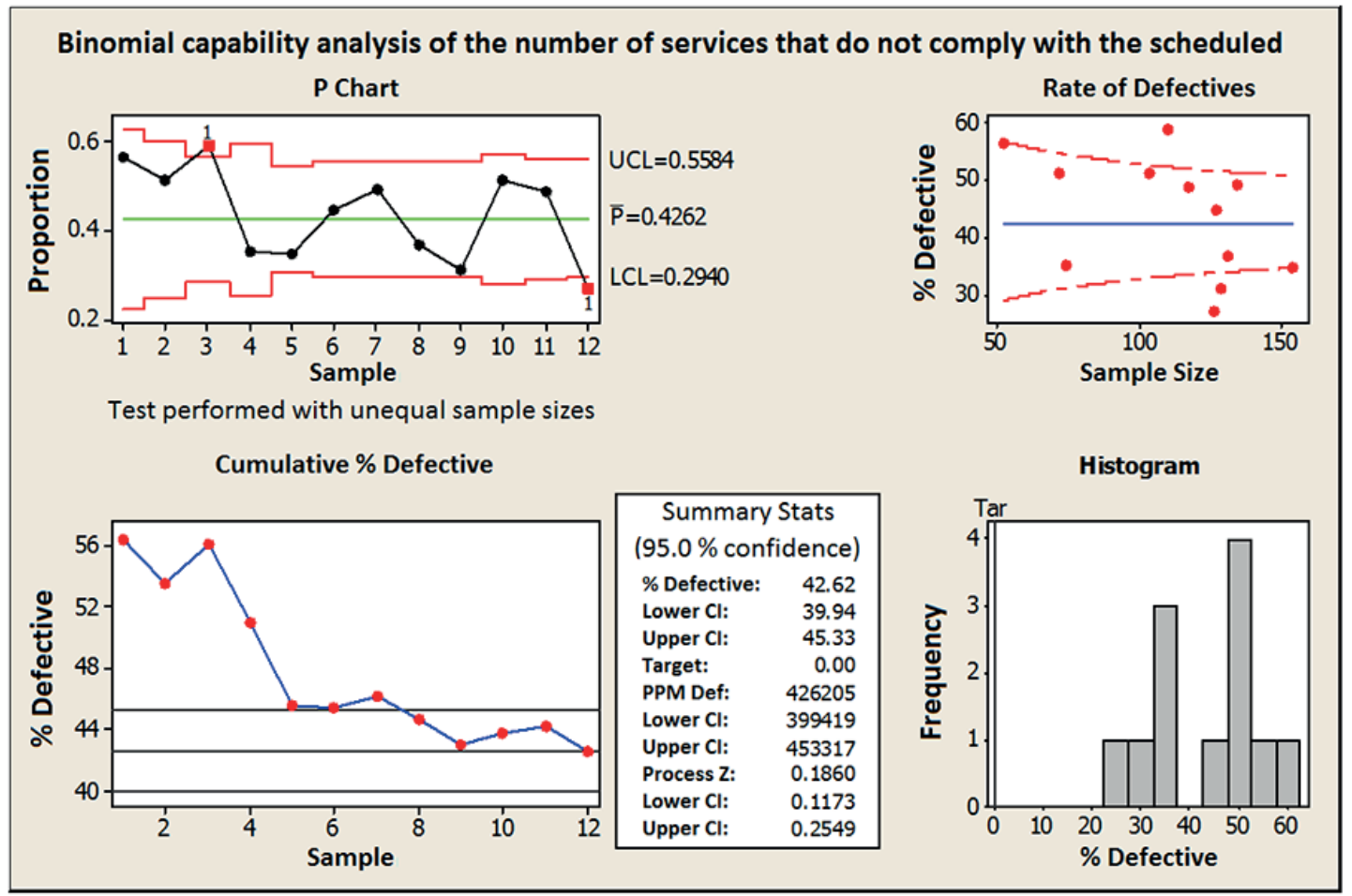

Figure 2. Binomial capability analysis of the number of services that do not comply with the scheduled date.

Source: Prepared by the author

software. Table 4 shows the result of the root cause analysis:

- Seller who creates the work order

- Lack of communication with the client

- Coordinator who schedules the calibration service

\section{Improve phase}

At this phase, the design and implementation of the solutions are established (Molteni \& Cecchi, 2005). In other words, action plans are determined to improve compliance with the scheduled dates, considering the participation of a multidisciplinary team, from which stands out:

- T field coordinator, who schedules the service date and examines the information that is collected in the work order and other considerations provided by the client when scheduling the service date.

- The seller, who creates the work order and provides the information related to the service to be provided.
- The lack of communication to the customer about the scheduled date of service.

In addition, in order to maintain and increase compliance with scheduled dates, mistake proofing mechanisms (Poka Yokes) are established, such as those shown in Table 5:

The variables (root cause) obtain an increase in compliance with the scheduled dates, as shown in Table 6, although not significant when the $p$-value $>0.05$. However, a decrease in variability is observed.

An increase in the level of customer satisfaction in meeting the scheduled dates of $84.6 \%$, compared to the initial $72.3 \%$, is observed.

\section{Control phase}

At this phase, the permanence of the results must be ensured (Molteni \& Cecchi, 2005). For this reason, the variables of the processes related to the scheduling and execution of the service are intended to maintain the improvements obtained. For this, the control plans in Table 7 are established: 
Table 4. Results of the analysis and root causes validation.

\begin{tabular}{|c|c|c|c|c|c|}
\hline Variable X & $\mathbf{Y}$ & $\begin{array}{l}\text { Statistics } \\
\text { technique }\end{array}$ & $p$-value & \begin{tabular}{|c|} 
Is it \\
significant? \\
\end{tabular} & Cause validation \\
\hline \multirow{2}{*}{$\begin{array}{l}\text { Seller who creates } \\
\text { the work order } \\
\text { (WO) }\end{array}$} & $\begin{array}{l}\text { Complies with } \\
\text { scheduled } \\
\text { date }\end{array}$ & $\begin{array}{l}\text { Two-way } \\
\text { ANOVA }\end{array}$ & 0.000 & Yes & \multirow{2}{*}{$\begin{array}{l}\text { The analysis is performed considering biweekly } \\
\text { data (X: seller; Y: complies with the scheduled } \\
\text { date). It is observed that, as they are not all nor- } \\
\text { mal distributions, Kruskal Wallis is applied and it is } \\
\text { found that the averages are significantly different } \\
\text { ( } p \text {-value of } 0.000)\end{array}$} \\
\hline & $\begin{array}{l}\text { Complies with } \\
\text { scheduled } \\
\text { date }\end{array}$ & $\begin{array}{l}\text { One-way } \\
\text { ANOVA }\end{array}$ & 0.000 & Yes & \\
\hline $\begin{array}{l}\text { Lack of commu- } \\
\text { nication with the } \\
\text { client }\end{array}$ & $\begin{array}{l}\text { Complies with } \\
\text { scheduled } \\
\text { date }\end{array}$ & $\begin{array}{l}\text { Correlation } \\
\text { and regression } \\
\text { analysis }\end{array}$ & 0.000 & Yes & $\begin{array}{l}\text { With the } 2 \text {-sample t-test statistic for the lack and } \\
\text { number of communications with the client, a } p \text {-va- } \\
\text { lue of } 0.014 \text { is obtained and it is concluded that it } \\
\text { affects compliance with scheduled date }\end{array}$ \\
\hline $\begin{array}{l}\text { The person who } \\
\text { schedules }\end{array}$ & $\begin{array}{l}\text { Complies with } \\
\text { scheduled } \\
\text { date }\end{array}$ & 2-sample t-test & 0.001 & Yes & - \\
\hline
\end{tabular}

Source: Prepared by the author.

Table 5. Poka-Yokes.

\begin{tabular}{|l|l|l|l|}
\hline \multicolumn{1}{|c|}{ Defects } & \multicolumn{1}{|c|}{ Errors } & Warning type & Level \\
\hline $\begin{array}{l}\text { Creating the work order without complete } \\
\text { technical information }\end{array}$ & Unawareness of sales staff & Warning & Detects error at the time \\
\hline $\begin{array}{l}\text { Failure to comply with the staff authorization } \\
\text { scheduling }\end{array}$ & Oversight/unawareness & Warning & Detects error at the time \\
\hline Programming non-authorized technical staff & Unawareness of the coordinator & Warning & Detects error at the time \\
\hline Scheduling staff that is not available & Mixed up dates due to inadvertences & Control & Detects error at the time \\
\hline $\begin{array}{l}\text { No calibration standards available for the } \\
\text { service }\end{array}$ & Oversight & Warning & Detects error at the time \\
\hline $\begin{array}{l}\text { Failure to inform the client of the service } \\
\text { scheduled date }\end{array}$ & $\begin{array}{l}\text { Oversight/unawareness/lack of cali- } \\
\text { bration standards }\end{array}$ & Warning & Eliminates source error \\
\hline Lack of service confirmation by the client & $\begin{array}{l}\text { Oversight/unawareness/lack of cali- } \\
\text { bration standards }\end{array}$ & Warning & Eliminates source error \\
\hline Delay in scheduling calibration service & Oversight/unawareness & Warning & Eliminates source error \\
\hline Reschedule the service & Oversight/unawareness & Warning & Eliminates source error \\
\hline $\begin{array}{l}\text { Failure to enter scheduled date into the } \\
\text { system }\end{array}$ & Oversight/unawareness & Warning & Detects error at the time \\
\hline Failure to inform technical staff & $\begin{array}{l}\text { Oversight/unawareness/lack of cali- } \\
\text { bration standards }\end{array}$ & Warning & Eliminates source error \\
\hline
\end{tabular}

Source: Prepared by the author.

Table 6. Results comparison of before and after the improvements implemented.

\begin{tabular}{|c|c|c|c|c|c|}
\hline \multirow[b]{2}{*}{ Variable } & \multicolumn{2}{|c|}{ Before improvements } & \multicolumn{2}{|c|}{ After improvements } & \multirow[b]{2}{*}{ p-value } \\
\hline & Mean & $\begin{array}{c}\text { Standard devi- } \\
\text { ation }\end{array}$ & Mean & $\begin{array}{c}\text { Standard devi- } \\
\text { ation }\end{array}$ & \\
\hline Coordinator 1 & $57.00 \%$ & 0.0522 & $68.88 \%$ & 0.0635 & 0.000 \\
\hline Coordinator 2 & $62.3 \%$ & 0.176 & $72.14 \%$ & 0.0674 & 0.091 \\
\hline Seller 1 & $54.80 \%$ & 0.115 & $62.95 \%$ & 0.0602 & 0.078 \\
\hline Seller 2 & $62.00 \%$ & 0.165 & $62.34 \%$ & 0.0595 & 0.943 \\
\hline Seller 3 & $61.0 \%$ & 0.189 & $62.80 \%$ & 0.0793 & 0.772 \\
\hline Seller 4 & $52.0 \%$ & 0.125 & $62.56 \%$ & 0.0503 & 0.040 \\
\hline Seller 5 & $50.2 \%$ & 0.201 & $62.96 \%$ & 0.0959 & 0.067 \\
\hline Seller 6 & $57.8 \%$ & 0.343 & $60.92 \%$ & 0.0483 & 0.763 \\
\hline Communication with the client & $64.4 \%$ & 0.146 & $77.71 \%$ & 0.0492 & 0.010 \\
\hline
\end{tabular}

Source: Prepared by the author. 
A comparative table of the indicators before and after applying the methodology was also made. The results are shown in Table 8.

From the above, although there has been an improvement in compliance with the scheduled date $(70.3 \%)$, this has not yet been sufficient to meet the minimum target of $80 \%$.

\section{DISCUSSION}

Specific hypothesis 1: The application of Six Sigma methodology positively impacts the compliance with scheduled dates for the execution of the calibration services in the field. To confirm the hypothesis, it was applied:

$$
\begin{aligned}
& \text { Ho: } \mu 1=\mu 2 \\
& \text { H1: } \mu 1 \neq \mu 2
\end{aligned}
$$

Where:

$\mu 1$ : The mean of the compliance with the scheduled dates of calibration services execution at the beginning.

$\mu 2$ : The mean of the compliance with the scheduled dates of calibration services execution at the end.

Therefore, in order to determine whether this variable improved, the before and after of the service

\begin{tabular}{|c|c|c|c|c|c|c|}
\hline $\begin{array}{l}\text { Process } \\
\text { step }\end{array}$ & Variable & Critical? & $\begin{array}{c}\text { Specification } \\
\text { (Characteristic to be } \\
\text { controlled) }\end{array}$ & Acceptance criteria & $\begin{array}{l}\text { Measurement } \\
\text { method }\end{array}$ & Control method \\
\hline \multirow{2}{*}{$\begin{array}{l}\text { Work order } \\
\text { review }\end{array}$} & $\begin{array}{l}\text { Technical informa- } \\
\text { tion on the work } \\
\text { order }\end{array}$ & Yes & \multirow{2}{*}{$\begin{array}{l}\text { Degree to which work } \\
\text { orders have com- } \\
\text { plete information on } \\
\text { the equipment to be } \\
\text { calibrated }\end{array}$} & \multirow{2}{*}{$\begin{array}{l}\text { Work orders with } \\
\text { complete technical } \\
\text { specifications on } \\
\text { the equipment to be } \\
\text { calibrated }\end{array}$} & \multirow{2}{*}{$\begin{array}{l}\text { Work orders } \\
\text { created }\end{array}$} & \multirow{2}{*}{$\begin{array}{l}\text { P-chart } \\
\text { Warning mechanism } \\
\text { to complete the } \\
\text { equipment data when } \\
\text { creating the proposal }\end{array}$} \\
\hline & Sales staff & Yes & & & & \\
\hline \multirow{4}{*}{$\begin{array}{l}\text { Service } \\
\text { scheduling }\end{array}$} & \multirow{2}{*}{ Planning } & Yes & $\begin{array}{l}\text { Degree of communica- } \\
\text { tion of the scheduled } \\
\text { service date }\end{array}$ & $\begin{array}{c}\text { Minimum } 75 \% \text { of } \\
\text { scheduled services }\end{array}$ & $\begin{array}{l}\text { Work order } \\
\text { control }\end{array}$ & $\begin{array}{l}\text { P-chart } \\
\text { Warning mechanism } \\
\text { to inform the custo- } \\
\text { mer of the date }\end{array}$ \\
\hline & & Yes & $\begin{array}{l}\text { Degree to which } \\
\text { services are scheduled } \\
\text { within two days after } \\
\text { creating the WO }\end{array}$ & $\begin{array}{l}\text { Minimum } 80 \% \text { of the } \\
\text { work orders created }\end{array}$ & $\begin{array}{l}\text { Work order } \\
\text { control }\end{array}$ & $\begin{array}{l}\text { P-chart } \\
\text { Error proofing system }\end{array}$ \\
\hline & Technical staff & Yes & $\begin{array}{l}\text { Compliance with the } \\
\text { personnel authoriza- } \\
\text { tion scheduling }\end{array}$ & $\begin{array}{l}\text { Personnel with valid } \\
\text { authorization }\end{array}$ & $\begin{array}{l}\text { Authorization } \\
\text { program }\end{array}$ & Error proofing system \\
\hline & $\begin{array}{l}\text { Technical staff } \\
\text { documents }\end{array}$ & No & $\begin{array}{l}\text { Maintain updated } \\
\text { personnel database, } \\
\text { considering the validity } \\
\text { of medical check-ups, } \\
\text { inductions, talks, etc. }\end{array}$ & $\begin{array}{l}\text { Technical staff that } \\
\text { meet customer } \\
\text { requirements }\end{array}$ & $\begin{array}{l}\text { Staff requi- } \\
\text { rements } \\
\text { database }\end{array}$ & Error proofing system \\
\hline \multirow{2}{*}{$\begin{array}{l}\text { Manage- } \\
\text { ment of } \\
\text { standards } \\
\text { delivery }\end{array}$} & \multirow{2}{*}{$\begin{array}{l}\text { Suitable for use } \\
\text { measurement } \\
\text { standards }\end{array}$} & \multirow{2}{*}{ Yes } & $\begin{array}{l}\text { Compliance with the } \\
\text { calibration and verifi- } \\
\text { cation of measurement } \\
\text { standards program }\end{array}$ & $\begin{array}{c}\text { Standards calibrated } \\
\text { and verified }\end{array}$ & $\begin{array}{l}\text { Calibration } \\
\text { and verifica- } \\
\text { tion program }\end{array}$ & $\begin{array}{l}\text { Error-proofing mech- } \\
\text { anism }\end{array}$ \\
\hline & & & $\begin{array}{l}\text { Compliance with the } \\
\text { measurement stan- } \\
\text { dards maintenance } \\
\text { program }\end{array}$ & $\begin{array}{l}\text { Maintained and ope- } \\
\text { rational standards }\end{array}$ & $\begin{array}{l}\text { Maintenance } \\
\text { program }\end{array}$ & $\begin{array}{l}\text { Error-proofing mecha- } \\
\text { nism }\end{array}$ \\
\hline \multirow{2}{*}{$\begin{array}{l}\text { Execution } \\
\text { of the } \\
\text { service }\end{array}$} & $\begin{array}{l}\text { Compliance with the } \\
\text { scheduled date }\end{array}$ & Yes & $\begin{array}{l}\text { Degree of compliance } \\
\text { with scheduled service } \\
\text { dates }\end{array}$ & $\begin{array}{l}\text { Minimum } 85 \% \text { of } \\
\text { services fulfilled }\end{array}$ & $\begin{array}{l}\text { Work order } \\
\text { control }\end{array}$ & $\begin{array}{l}\text { P-chart } \\
\text { Warning mechanism }\end{array}$ \\
\hline & $\begin{array}{l}\text { Customer satisfac- } \\
\text { tion }\end{array}$ & Yes & $\begin{array}{l}\text { Very good result in the } \\
\text { customer satisfaction } \\
\text { survey }\end{array}$ & $\begin{array}{l}\text { Minimum } 80 \% \text { satis- } \\
\text { faction }\end{array}$ & $\begin{array}{l}\text { Customer } \\
\text { satisfaction } \\
\text { surveys }\end{array}$ & Sampling \\
\hline
\end{tabular}

Table 7. Control plan.

Source: Prepared by the author. 
Table 8. Comparative table of before and after the methodology use.

\begin{tabular}{|l|c|c|}
\hline \multicolumn{1}{|c|}{ Indicator } & Before \\
\hline Management indicators & 0.356 & 0.348 \\
\hline PC & Not capable and unstable process & Not capable and not centered process \\
\hline SPC and capacity & $42.74 \%$ & $29.44 \%$ \\
\hline$\%$ defective & 427376 & 294360 \\
\hline DPMO & 0.1831 & 0.5407 \\
\hline Process Z & 1.68 & 2.04 \\
\hline Sigma level & $56.2 \%$ & $70.3 \%$ \\
\hline Central Limit-Compliance with the scheduled date & S/. 747230 & S/. 463 989 \\
\hline Variable costs & & $84.6 \%$ \\
\hline Quality indicators & $72.3 \%$ & 10 \\
\hline Customer Satisfaction-Compliance with the scheduled date & 31 & $19.6 \%$ \\
\hline Complaints received related to scheduling & $31.4 \%$ & \\
\hline Complaints due to scheduling noncompliance & & \\
\hline
\end{tabular}

Source: Prepared by the author.

execution scheduled date were compared, by applying the two-sample t-test.

Figure 3 shows that, since the confidence interval is between $<-0.2135 ;-0.0701>$, the reference value of 0 is not found within it and that the $p$-value $(0.001)$ $<\alpha(0.05)$. Then, $\mathrm{Ho}$ is rejected with a $95 \%$ level of confidence.

Therefore, it was concluded that the compliance with the scheduled dates of the calibration services execution before and after the application of the methodology is different.

Specific hypothesis 2: The identification of the factors that have the greatest influence on the compliance with the service execution scheduled dates has a positive impact on the level of customer satisfaction after implementing the Six Sigma methodology. To confirm the hypothesis, it was applied:

$$
\begin{gathered}
\text { Ho: } p(1)-p(2) \\
H 1: p(1)-p(2) \neq 0
\end{gathered}
$$

Where:

$p(1)$ : Proportion of dissatisfied customers before improvements were implemented.

$p(2)$ : Proportion of dissatisfied customers after improvements were implemented.

Therefore, to determine whether there was improvement on this variable, the before and after level of customer satisfaction was compared, by applying the two-test.

Figure 4 shows that, since the confidence interval is between $<0.0242658 ; 0.221888>$, the reference value of 0 is not found within it. In addition to the $p$-value $(0.015)<\alpha(0.05), \mathrm{Ho}$ is rejected with a $95 \%$ level of confidence.

It was concluded that there is a difference between customer satisfaction with respect to compliance with the scheduled service dates before and after implementing the improvements with the Six Sigma methodology.

Specific hypothesis 3: The implementation of actions on the factors that influence compliance with the scheduled date positively impacted the level of complaints received associated with the services scheduling. To confirm the hypothesis, it was applied:

$$
\begin{aligned}
& \text { Ho: } \mu 1=\mu 2 \\
& \text { H1: } \mu 1 \neq \mu 2
\end{aligned}
$$

Where:

$\mu 1$ : The average ratio of complaints received for scheduling services at the beginning.

$\mu 2$ : The average ratio of complaints received for scheduling services at the end.

Therefore, in order to determine whether this variable improved, the before and after of the average 


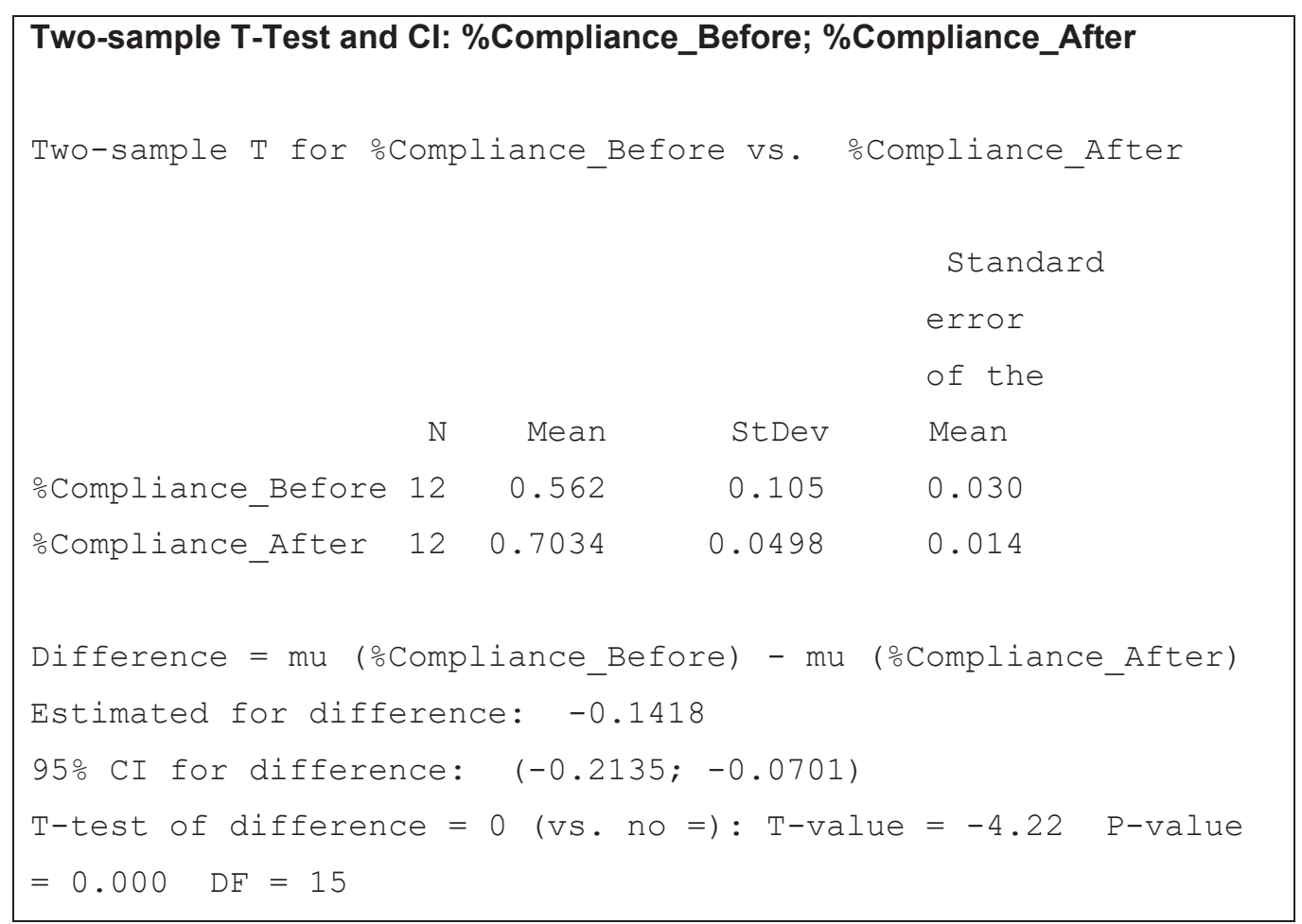

Figure 3. Two-sample $\mathrm{T}$ and $\mathrm{Cl}$ test: compliance of scheduled dates for calibration services. Before and after implementing Six Sigma methodology. Source: Prepared by the author



Figure 4. Test for two ratios: level of customer satisfaction due to compliance with scheduled dates. Before and after implementing Six Sigma methodology.

Source: Prepared by the author. 
ratio of the complaints received related to the service scheduling were compared, by applying the two-sample t-test.

Figure 5 shows that, since the confidence interval is between $<-0.0114 ; 0.2470>$, the reference value of 0 is found within it. In addition to the $p$-value $(0.067)>\alpha(0.05), \mathrm{Ho}$ is accepted with a $95 \%$ level of confidence.

Therefore, it was concluded that the ratio of complaints received for service scheduling at the beginning of the project and the ratio of complaints received for service scheduling after the methodology was implemented are the same.

\section{RECOMMENDATIONS}

- Raise awareness among senior management regarding the importance of their participation in the project, in order to guarantee resources and management support and to impact improvement towards the organization and, mainly, the customer.

- Maintain fluent communication with the staff in charge of organizing the project development, in order to guarantee trust, participation and commitment in order to improve.

- Maintain the training program for the staff involved, directly or indirectly, with the improved process.

- Use the Six Sigma methodology to comply with the delivery of the calibration certificates, whose scope is the processing of the calibration records, issuing the calibration certificates and delivering the certificates to the client.

\section{CONCLUSIONS}

- The application of Six Sigma methodology positively impacts the compliance with scheduled dates for the execution of the calibration services in the field, increasing compliance from $56.2 \%$ to $70.34 \%$.

- The identification of the factors of greatest influence on the compliance with the service execution scheduled dates had a positive impact on the level of customer satisfaction after implementing the Six Sigma methodology, increasing compliance from $72.3 \%$ to $84.6 \%$.



Figure 5. Two-sample T-Test and Cl: ratio of complaints received for service scheduling. Before and after implementing Six Sigma methodology.

Source: Prepared by the author 
- The implementation of actions on the factors that influence compliance with the scheduled date positively impacted the level of complaints received associated with the services scheduling, decreasing complaints from $31.4 \%$ to $19.63 \%$.

\section{REFERENCES}

[1] Besterfield, D. H. (2009). Control de calidad. México D. F., Mexico: Pearson Educación.

[2] Desai, D. A. (2006). Improving Customer Delivery Commitments the Six Sigma Way: Case Study of an Indian Small Scale Industry. International Journal of Six Sigma and Competitive Advantage, 2(1), 23-47.

[3] Evans, J. R. \& Lindsay, W. M. (2008). Administración y control de la calidad. México D. F., Mexico: Cengage Learning.

[4] Gryna, F. M. (2002). Investigación de mercados y marketing. In J. M. Juran \& A. B. Godfrey (Coords.), Manual de calidad de Juran (tomo 1) (pp. 18.1-18.38). Madrid, Spain: McGraw-Hill.

[5] Gutiérrez, H. (2008). Los retos de la mejora de la calidad y la productividad en las organizaciones. Ingeniería Industrial. Actualidad y Nuevas Tendencias, 1(1), 109-124.

[6] Hernández, R., Fernández, C. \& Baptista, P. (2010). Metodología de la investigación. México D. F., Mexico: McGraw-Hill.

[7] Lankford, R. (2005). Programación de la producción. En K. Zandin, Maynard: Manual del ingeniero industrial (pp. 9.157-9.176). México D. F., Mexico: McGraw-Hill.

[8] Molteni, R. \& Cecchi, O. (2005). El liderazgo del Lean Six Sigma. Buenos Aires, Argentina: Macchi.

[9] Nakhai, B. \& Neves, J. S. (2009). The Challengues of Six Sigma in Improving Service Quality. International Journal of Quality \& Realiability Management, 26(7), 663-684.
[10] Oke, S. A. (2007). Six Sigma: A Literature Review. South African Journal of Industrial Engineering, 18(2), 109-129.

[11] Organización Internacional de Normalización (2015). Norma internacional ISO 9000. Sistemas de gestión de la calidad. Fundamentos y vocabulario. Geneva, Switzerland: Secretaría General de ISO.

[12] Palacio, V., Quispe, F., Ylesca, F., Cañi, E. \& Velazco, C. (2013). Rediseño y optimización de procesos de CERPER. Lima, Perú: Instituto para la Calidad de la Pontificia Universidad Católica del Perú.

[13] Pérez, D. A. (2014). Cultura del incumplimiento empresarial en México. Ensayo. Retrieved from http://www.gestiopolis.com/culturadel-incumplimiento-empresarial-en-mexicoensayo.

[14] Quijano, V. M. (2003). Cumplimiento del servicio prometido a sus clientes. Retrieved from http://www.gestiopolis.com/cumplimientoservicio-prometido-clientes.

[15] Ramos, W. A. (2013). Incremento de la productividad a través de la mejora continua en calidad en la subunidad de procesamiento de datos en una empresa courier: el caso Perú Courier. Industrial Data, 16(2), 59-66.

[16] Rojas, L. C. (2008). Implementación del sistema de gestión de calidad según la Norma ISO 9001:2000 en una industria plástica. (Degree thesis). Escuela Superior Politécnica del Litoral, Guayaquil.

[17] Sánchez, H. \& Reyes, C. (2015). Metodología y diseños en la investigación científica. Lima, Peru: Business Support Aneth S. R. L. 